3

Contact information of the authors

\begin{tabular}{|c|c|}
\hline Name & Email address \\
\hline Qingli Guo ${ }^{1,2,3}$ & qingli.guo@helsinki.fi \\
\hline Eszter Lakatos $^{2}$ & e.lakatos@qmul.ac.uk \\
\hline Ibrahim Al Bakir ${ }^{2}$ & i.albakir@qmul.ac.uk \\
\hline Kit Curtius² & k.curtius@qmul.ac.uk \\
\hline Trevor A. Graham ${ }^{2, *}$ & t.graham@qmul.ac.uk \\
\hline Ville Mustonen 1,3,* & v.mustonen@helsinki.fi \\
\hline
\end{tabular}

* correspondence to t.graham@qmul.ac.uk or v.mustonen@helsinki.fi

\title{
The mutational signatures of formalin fixation on
} the human genome

Qingli Guo ${ }^{1,2,3}$, Eszter Lakatos², Ibrahim Al Bakir², Kit Curtius², Trevor A. Graham²,*, Ville Mustonen ${ }^{1,3, *}$ 1. Organismal and Evolutionary Biology Research Programme, Department of Computer 2. Evolution and Cancer Laboratory, Centre for Genomics and Computational Biology, Barts Cancer Institute, Barts and the London School of Medicine and Dentistry, Queen Mary University of London,

3. Institute of Biotechnology, Helsinki Institute for Information Technology, University of Helsinki, 00014 Helsinki, Finland. 


\section{Abstract}

\section{Background}

18 Formalin fixation and paraffin embedding (FFPE) of patient material remains standard practice in

19 clinical pathology labs around the world. Clinical archives of patient material near-exclusively consist of FFPE blocks. The ability to perform high quality genome sequencing on FFPE-derived DNA would accelerate a broad spectrum of medical research. However, formalin is a recognised mutagen and sequencing of DNA derived from FFPE material is known to be riddled with artefactual mutations.

\section{Results}

24 Here we derive genome-wide mutational signatures caused by formalin fixation, and provide a computational method to correct mutational profiles for these formalin-induced artefacts. We show that the FFPE-signature is dominated by $\mathrm{C}>\mathrm{T}$ transitions caused by cytosine deamination, and has very high similarity to COSMIC signature SBS30 (base excision repair deficiency due to inactivation mutations in NTHL1). Further, we demonstrate that chemical repair of formalin-induced DNA lesions, a process that is routinely performed as part of sequencing library preparation, leads to a signature highly similar to COSMIC signature SBS1 (spontaneous deamination of methylated cytosine). Next, we design FFPEsig, a computational method to remove the formalin-induced artefacts from mutational counts. We prove the efficacy of this method by generating synthetic FFPE samples using 2,780 cancer genomes from the Pan-Cancer Analysis of Whole Genome (PCAWG) project, and via analysis of FFPEderived genome sequencing data from colorectal cancers.

\section{Conclusions}

Formalin fixation leaves a predictable mutational footprint across the genome. The application of our FFPEsig software corrects the mutational profile for the influence of formalin, enabling robust mutational signature analysis in FFPE-derived patient material. 


\section{Keywords}

41 Formalin fixation paraffin embedding (FFPE), mutational signatures, artefact correction, computational genomics

\section{Background}

Patient samples are routinely processed with formalin fixation and paraffin embedding (FFPE) by pathology laboratories around the world. FFPE preserves tissue morphology and enables immunohistochemical analysis for clinical diagnosis [1,2]. However, genomic analysis of DNA extracted from FFPE blocks is problematic, as formalin fixation negatively impacts DNA quantity and quality compared to fresh frozen (FF) material $[3,4]$. The pathology archive of any large hospital is likely to contain tens of thousands of FFPE blocks. Enabling accurate genomic analysis of FFPE material would unlock the tremendous translational research potential of these vast collections of archival material.

During fixation step of FFPE preservation, buffered formalin ( $4 \%$ formaldehyde) penetrates the biospecimen and generates cross-links between intracellular macromolecules (DNA-DNA, DNA-RNA and DNA-protein). These crosslinks stall DNA polymerases during library amplification [5-7]. As a consequence, the diversity and the number of templates that can be amplified by PCR from FFPE DNA is significantly depleted [4,8]. Furthermore, formalin causes hydrolytic deamination of cytosine bases to uracil $[1,5]$, resulting in $\mathrm{U}: \mathrm{G}$ mismatches where DNA polymerase incorporates adenine opposite to uracil in amplicon-based protocols, generating artefactual $\mathrm{C}: \mathrm{G}>\mathrm{T}: \mathrm{A}$ substitutions in sequencing data $[5,9,10]$

To mitigate deamination artefacts, some FFPE sequencing library preparations include "repair treatment" whereby uracil DNA glycosylase (UDG) is added to remove uracil bases prior to amplification [9-11]. However, for 5-methylcytosine $(5 \mathrm{mC})$ in $\mathrm{CpG}$ dinucleotides, deamination by 
66 formalin would be converted directly to thymine instead of uracil $[3,8]$. This second class of formalin

67 artefact is not corrected by the repair treatment therefore, downstream bioinformatics approaches are necessary to attempt their removal [5].

69

70 Mutational signatures derived from whole genome sequencing (WGS) data characterise the mutational processes that have acted upon the DNA within a sample [12,13], and they hold tremendous potential for diagnosis and therapeutic guidance [14-18]. Single base substitution (SBS) signatures are derived by considering the type of specific base pair change (e.g. $\mathrm{C}>\mathrm{T}$ or $\mathrm{C}>\mathrm{A}$, etc.) together with the flanking base pair context (e.g. ACA $>$ ATA, or ACA $>$ AAA, etc.) $[12,13]$. The recently updated mutational signature catalogue provides a comprehensive source of mutational processes active in human cancers that is derived from an unprecedentedly large number of samples [19]. As the artefactual mutations from FFPE preservation will bias mutational profiles, they have to be taken into account when unravelling mutational processes from FFPE samples.

Here, we use the statistical machinery of mutational signature analysis to derive mutational footprint caused by formalin exposure during FFPE biospecimen processing. First, we identify the "formalin artefact" mutational signatures in both unrepaired and repaired FFPE samples, using paired FFPE and FF sequencing data from the same samples. We next design and validate a decomposition algorithm,

84 FFPEsig, to subtract FFPE artefacts and thereby infer mutational profiles of biological origin in genome sequencing data from an FFPE specimen. Our method enables robust mutational profile correction of FFPE samples for research and potential clinical implementation. 


\section{$87 \quad$ Results}

\section{Mutational signatures of formalin fixation}

\section{Formalin fixation artefacts are predominantly $\mathrm{C}>\mathrm{T}$ mutations}

90 To identify artefacts signatures, we used publicly available targeted panel sequencing data from two previous studies $[8,11]$, in which triplicate samples (FFPE-repaired, FFPE-unrepaired and FF) were available. The study by Prentice et al. (hereafter study 1 ) comprised colorectal cancers $(n=3)$, and each cancer included nine samples: one FF sample, four unrepaired and four repaired FFPE samples that were sequenced after a fixation time of 2, 15, 24 and 48 hours respectively. In addition, study 1 included patients $(n=29)$ for whom repaired and unrepaired FFPEs were available. In the study by Bhagwate et al. (hereafter study 2), triplicate samples from benign breast tissue $(n=4)$ were available. In total, we obtained 110 FFPE samples, of which 32 (29\%) had matched FF (see Methods \& Materials).

98

We first focused on samples with matched FF available, and examined the set of mutations detected in FFPE samples but not detected in matched FF samples (termed FFPE-only or discordant mutations). Within the study 1 sample set, we discovered that $\mathrm{C}>\mathrm{T}$ discordant mutations were common $(45.8 \%$ and $21.1 \%$ in unrepaired and repaired samples, respectively). $\mathrm{T}>\mathrm{C}$ mutations were also common $(53.5 \%$ and 76.3\% in unrepaired and repaired FFPEs, respectively; Supplemental Fig 1). Discordant FFPE-only mutations from study 2 also tended to be $\mathrm{C}>\mathrm{T}$ mutations $(98.9 \%$ in unrepaired and $76.6 \%$ in repaired FFPEs), but very few $\mathrm{T}>\mathrm{C}$ mutations were detected in this second study $(0.55 \%$ in unrepaired and $11.6 \%$ in repaired FFPEs; Supplemental Fig 2).

To examine whether $\mathrm{T}>\mathrm{C}$ mutations were true artefacts of FFPE, we counted the proportion of $\mathrm{C}>\mathrm{T}$ and $\mathrm{T}>\mathrm{C}$ mutations present in two or more of the set of samples from a patient ('concordant mutations'). On average, about $30 \% \mathrm{C}>\mathrm{T}$ mutations were shared by at least two samples, in contrast to $88 \%$ for $\mathrm{T}>\mathrm{C}$ mutations (Supplemental Fig 3a). We next compared frequencies of concordant mutations between all sample-pairs across three patients: $12 \%$ of $\mathrm{C}>\mathrm{T}$ mutations and $59 \%$ of $\mathrm{T}>\mathrm{C}$ mutations were shared by 
113 one sample-pair on average (Supplemental Fig 3b). Furthermore, $\mathrm{C}>\mathrm{T}$ discordant mutation loads

114 increased with formalin fixation time in both repaired (slope $=0.80$, intercept $=89.68$ ) and unrepaired

115 FFPE samples (slope $=7.48$, intercept=164.81) (Fig 1a). However, the $\mathrm{T}>\mathrm{C}$ discordant mutation loads

116 decreased with fixation time in unrepaired FFPE (slope $=-0.63$, intercept $=350.85$ ), but increased in

117 repaired FFPEs (slope=1.02, intercept=364.62) (Supplemental Fig1). Taken together, our results

118 suggested that $\mathrm{C}>\mathrm{T}$ mutations are the predominant true formalin induced artefacts, and that $\mathrm{T}>\mathrm{C}$

119 mutations are likely caused by other sources of mutational noise rather than formalin fixation.

120 Unrepaired formalin signature is highly similar to SBS30; repaired formalin 121 signature is highly similar to SBS1

122 We next used all FFPE-only mutations ( $\mathrm{T}>\mathrm{C}$ excluded) to learn FFPE signatures. Analysis was performed on all FFPE samples $(\mathrm{n}=110)$. The samples in the respective studies were sequenced using different cancer gene panels, thus the 'mutational opportunities', determined by the frequency of each trinucleotide context in the panel, differed between studies (Supplemental Fig 4). Therefore, we applied the study-specific normalisation on the mutation counts to enable direct comparison between the studies (see Methods \& Materials). The cluster of normalised mutational profiles from the entire combined set of $n=110$ FFPE samples was represented using t-distributed stochastic neighbour embedding (t-SNE) [20] (Fig 1b). Samples from the two studies showed no batch effect and clearly separated into two clusters of unrepaired and repaired samples. A single repaired sample from study 1 clustered with unrepaired FFPEs, which we suspect is due to poor response to UDG treatment [21]. In addition, we clustered $\mathrm{T}>\mathrm{C}$ mutational profiles after normalisation, but discovered a clear batch effect and found no consistent error patterns (Supplemental Fig 3c).

To exclude possible outliers, we used t-SNE clustering to select representative samples. We performed an iterative process where each iteration was defined by the random seed inputted to the t-SNE algorithm. For each t-SNE embedding, we calculated the spatial density of the clustered data measured by a gaussian kernel, and selected samples in regions of high density (density $>0.018$ ) as our representative sample subset (Supplemental Fig 5a). The averaged values of all mutation channels from 
this representative subset generated one set of FFPE signature candidates. Our final FFPE signatures were derived from the mean of 100 candidates collected from 100 t-SNE embeddings (Supplemental

142 Fig 5b and 5c; Supplemental Table 1).

144 We then compared the derived FFPE artefact profiles to the latest COSMIC SBS signatures (V3 - May 2019) [19] (Fig 1c), and found that unrepaired and repaired FFPE signatures are highly similar to SBS30 and SBS1 respectively (cosine similarity 0.90 for both; Fig 1d and 1e). SBS30 has been validated as a mutational footprint of NTHL1 mutations that disrupt base excision repair (BER) [22,23]. SBS1 is wellknown as a 'clock-like' signature that positively correlates with patient age, as a consequence of spontaneous deamination of methylcytosine [24]. We note that the unrepaired FFPE signature shared even greater similarity with COSMIC V2 (March 2015) signature 1 (0.95), which was inferred from a smaller cohort compared to SBS1 of V3.

Despite the high similarity, there were certain mutation channels that differed between FFPE signatures and the two known mutational processes. We marked the mutation channels if the fold-change was over 2 (Fig 1d and 1e). Unrepaired FFPE signature differs in NCT context. The repaired FFPE signature mostly differs in non-CpG mutation channels which are absent in SBS1(V3) but present in sig 1 (V2). Those small proportions of mutations in non-CpG channels of repaired FFPE signature are likely due to the artefactual mutations escaped from the UDG repairing process.

\section{Development and validation of FFPE artefacts correction algorithm}

\section{0 using synthesised data}

161 We designed and implemented an algorithm we called "FFPEsig" to correct artefacts from FFPE mutational profiles (see Methods \& Materials). The algorithm decomposes the observed aggregate mutational catalogue of one given FFPE sample as the combination of FFPE-artefacts and the true biological mutations. To test the performance of the method, we added FFPE-artefacts to all PCAWG 
true, simulated and corrected profiles for one colorectal cancer (CRC) sample. In this case, FFPEsig successfully inferred the biological mutation catalogue with $\sim 0.99$ accuracy, measured by cosine similarity on $\mathrm{C}>\mathrm{T}$ channels. The correction accuracy was slightly higher when we used the full 96channel (Supplemental Fig 6), but the predominance of formalin associated mutations in the $\mathrm{C}>\mathrm{T}$ channels meant the gain was minimal. Therefore, hereafter we evaluated our correction accuracy focusing only on $\mathrm{C}>\mathrm{T}$ mutation channels.

Overall, FFPEsig achieved 0.89 mean correction accuracy for both unrepaired (95\% CI: $0.885,0.893$ ) and repaired FFPEs (95\% CI: 0.887, 0.894) (Fig 2b and 2c). To examine the possible factors which could influence the artefact correction, we evaluated 1) biological mutation count; 2) the similarities between the artefact signature (the 'noise') and the true biological mutation catalogue (the 'signal'). Poorly corrected cases were due to low mutation load and/or high similarity of patterns shared between the noise and signal (Fig 2b). We noticed that samples with low biological mutation load were difficult to correct regardless of how different the mutation patterns are from the FFPE signatures (purple dots in Fig $2 b)$. We further separated these two factors and confirmed that higher biological mutation burden led to more accurate correction (Fig 2d), as well as high dissimilarity between the signal and the noise (Fig 2e; cases with low mutation load excluded).

We continued our in silico evaluation by examining correction performance across cancer types for simulated unrepaired and repaired FFPEs within each cancer type (Fig 2c). The efficacy of correction varied significantly across 26 cancer types. FFPEsig was most accurate in skin melanoma (mean: 0.98 ) due to its high mutation load (96,361 SBSs) and low similarity to the noise signatures $(0.55)$ for both FFPE samples, followed by bladder transitional cell carcinoma (Bladder-TCC, 0.97) and lung squamous cell carcinoma (Lung-SCC, 0.96). In contrast, FFPEsig performed poorly for pilocytic astrocytoma (CNS-PiloAstro, 0.61), thyroid adenocarcinoma (Thy-AdenoCA, 0.80) and medulloblastoma (CNSMedullo, 0.82), because of the low averaged mutation loads (from 112 to 602) and relatively higher similarity to the noise signatures $(0.69-0.74)$ in these cancer types. 
194 We also noticed that the algorithm had different performance between unrepaired and repaired FFPEs

195 within certain cancer types. There were 17 out of 26 cancer types with detectable difference in 196 correction efficacy ( $p$-value $<0.05)$ and 12 of 17 with a highly significant difference $(p$-value $<0.001)$.

197 For instance, the correction worked much better in unrepaired FFPEs for colorectal (ColoRect198 AdenoCA) and pancreatic adenocarcinoma (Panc-AdenoCA), with 98\% and 92\% of well-corrected 199 samples for unrepaired FFPEs respectively, in contrast to only $71 \%$ and $51 \%$ respectively for repaired ones. Since the mutation burdens were the same for two types of FFPEs within a cancer type, the 201 significant difference is caused by true mutations being more dissimilar to the FFPE-artefact profile in 202 unrepaired FFPEs (cosine similarity 0.49 for CRCs and 0.59 for pancreatic cancers), whereas the 203 repaired-FFPE mutational signature was very similar to the true mutational profile (cosine similarity 2040.89 and 0.90 colorectal and pancreatic cancer respectively). By contrast, FFPEsig worked successfully in repaired-FFPEs for Lung-SCC and liver hepatocellular carcinoma (Liver-HCC), with 100\% and 96\% well-corrected samples for the opposite reason.

208 Finally, we explored how the accuracy of FFPE-artefact removal changes with increasing noise of FFPE 209 artefacts (Fig 2f). We selected four cancer types with $80 \%$ or more well-corrected samples in both 210 repaired and unrepaired FFPEs, including 219 tumour samples from CNS-GBM, Skin-Melanoma, 211 Bladder-TCC and Lung-SCC (Fig 2c). As expected, as the burden of artefactual mutations was increased, the correction accuracy dropped from 0.97 to 0.86 in unrepaired FFPEs, and from 0.98 to

2130.84 in repaired FFPEs. Overall, FFPEsig performed equally well in both types of FFPE with up to $10^{5}$ 214 noise (mean accuracy $>0.94$ ), but its performance dropped dramatically for samples with $10^{6}$ noise 215 (0.84-0.86). Thus, our method works for samples that hold reasonable signal-to-noise ratio, but not for 216 the extreme cases, e.g. samples with $10^{6}$ noise in this experiment with signal-to-noise ratio around $217 \quad 0.0088$. 


\section{A case study of correcting FFPE artefacts in WGS FFPE CRC blocks 219 shows consistent results with simulated data}

220 Next, we performed whole genome sequencing on two tumour FFPE samples (unrepaired versus repaired), and on the normal tissue DNA as matched normal from the same CRC patient (see Methods $\&$ Materials; FF material was not available). The mean coverages of the sequencing data were $46 \mathrm{X}$ (unrepaired FFPE), 43X (repaired FFPE) and 43X (normal sample), with 98.81\% or more of reads mapped to the genome (Supplemental Table 2). Following filtering (see Methods \& Materials), we detected 13,208 and 6,107 somatic single base substitutions in unrepaired and repaired FFPE, respectively (Supplemental Fig 7a and 7b). In particular, the two types of dominant mutations in our FFPE samples were $\mathrm{C}>\mathrm{T}$ and $\mathrm{T}>\mathrm{C}$, and together they contributed $64.7 \%-66.6 \%$ to the total mutations (Supplemental Fig $7 b$ ). For $\mathrm{C}>\mathrm{T}$ mutations, we expected them to be a mixture of FFPE artefacts and real biological mutations, because of the relative preponderance $(\sim 35 \%)$ of $\mathrm{C}>\mathrm{T}$ mutations in PCAWG CRCs. T $>$ C mutations accounted for $41.2 \%$ and $39.8 \%$ in our unrepaired and repaired FFPEs, but only $\sim 16 \%$ in PCAWG CRCs (Supplemental Fig 7c). Similarly, large proportions of T $>$ C mutations were also detected in FFPE samples in study 1 (Supplemental Fig 1). As noted above, these presumably artefactual $\mathrm{T}>\mathrm{C}$ mutations did not show consistent patterns (Supplemental Fig 3c). Therefore, we excluded $\mathrm{T}>\mathrm{C}$ mutations from further study.

Since matched FF was not available to provide the ground truth mutational signature, we were inspired by results found in study 2 [8], where both repaired and unrepaired FFPE samples contained the majority of the variants found in the matched FF sample. Thus, we used concordant mutations with more strict filtering (variant supporting reads $\geq 5$ in both FFPEs) as an approximation for the true biological mutation profile of the tumour: this yielded a total of 1040 filtered concordant mutations (Supplemental Fig $7 \mathrm{a}$ and $7 \mathrm{~b}$ ), and 656 of them remained after excluding $\mathrm{T}>\mathrm{C}$ mutations (top panel of Fig $3 \mathrm{a}$ ).

243 To obtain more general knowledge about the biological mutation profiles of CRCs, we performed 244 hierarchical clustering on the 60 PCAWG CRC samples and discovered the samples share highly 
245 homologous mutational profiles within each subtype, namely MSS, MSI and POLE (Supplemental Fig

246 8a). The averaged sample-pair cosine similarity is 0.90 for MSS-CRCs, 0.92 for MSI-CRCs and 0.96

247 for POLE-CRC, but profiles between subtypes are significantly different (Supplemental Fig 9a). To

248 identify the most "conserved" mutation patterns within each subtype, we performed a similar analysis

249 on six mutation types separately, which showed $\mathrm{C}>\mathrm{A}$ and $\mathrm{C}>\mathrm{T}$ mutations have the strongest power in 250 classifying CRC subtypes (Supplemental Fig $8 b$ and 9b). Therefore, we compared the concordant C $>$ A mutations observed in our case to the PCAWG CRCs and identified that our sample was a MSS-CRC (Fig 3b).

We next applied FFPEsig on the observed mutation counts from the two FFPE samples and valuated the corrected profiles (Fig 3a and Supplemental Fig 10) by comparing them to concordant mutation catalogue as well as all PCAWG MSS-CRC samples, under the assumption that after removing artefacts the mutational profile of our samples should show higher similarity to both 'positive controls'. For unrepaired FFPE CRC, the accuracy improved from 0.906 before correction to 0.945 after correction to concordant mutations (Fig 3c). When compared to MSS-CRCs, the correction led to a significant increase in cosine similarity from 0.841 to 0.918 (Fig 3d). However, correction on repaired FFPE CRC generated the opposite results (Fig $3 \mathrm{c}$ and $3 \mathrm{~d}$ ). We validated our observations using simulated FFPE MSS-CRCs and confirmed that the correction was only beneficial for unrepaired not repaired FFPEs (Fig 3e). This was because the biological MSS-CRC profiles are highly similar to the repaired FFPE signature (0.98 on $\mathrm{C}>\mathrm{T}$ channels) and so our correction method could not distinguish true mutations from artefacts.

We further investigated how our corrected profile from unrepaired FFPE could contribute to CRC subtyping. Application of MSIsensor [26] detected 8.3\% of microsatellite sites with somatic changes in the unrepaired FFPE sample, but only $0.23 \%$ from the repaired FFPE. $8.3 \%$ exceeds the $3.5 \%$ threshold to call MSI [26], and so application of MSIsensor to an unrepaired FFPE sample could lead to miscalling of MSI status. We therefore attempted to classify the sample using the 'conserved' mutation patterns within CRC subtypes (described above). The unrepaired FFPE sample was equally similar to both using 
observed $\mathrm{C}>\mathrm{A}$ and $\mathrm{C}>\mathrm{T}$ trinucleotide mutational counts together or only $\mathrm{C}>\mathrm{T}$ mutations (Supplemental

274 Fig 11a and 11b). However, following correction using FFPEsig, we could clearly distinguish that the 275 sample was MSS. In addition, we found that the C>A mutation pattern itself could also classify our sample (Supplemental Fig 11c). As FFPEsig mostly in $\mathrm{C}>\mathrm{T}$ channels, $\mathrm{C}>\mathrm{A}$ patterns were almost the same with or without correction (0.99).

\section{8}

Potential of using 80-channel signatures for refitting analysis in FFPE samples

$\mathrm{T}>\mathrm{C}$ were common in some but not all FFPE samples in our dataset, and perhaps resulted in differences in sequencing library preparation methodology between studies. To attempt to control for this unexplained variation, here we examined the impact of removing all $\mathrm{T}>\mathrm{C}$ variants during signature refitting analysis. We compared the attributed mutation count (or activity) of each signature by supplying our refitting model with 80-channel (80c; $\mathrm{T}>\mathrm{C}$ removed) and 96-channel (96c) signatures on PCAWG mutational catalogues (see Methods \& Materials; Supplemental Fig 12). The $\log _{10}$ signature activity ratio of $80 \mathrm{c}$ to $96 \mathrm{c}$ was used to estimate how consistent both results were, and we termed this value as an inconsistency rate. The bigger the absolute inconsistency rate is, the more different the attributions are.

We refitted 10,312 mutational signature activities for 29 active signatures from 2,726 PCAWG genomes (Fig 4a), and an additional 54 genomes were excluded from original PCAWG dataset due to either low reconstruction accuracy $(<0.85 ; \mathrm{n}=35)$ by $96 \mathrm{c}$ signatures or too small of a sample size $(<10$ cases per signature per cancer type; $\mathrm{n}=19)$. The mean inconsistency rate among 10312 refits was $0.013(95 \% \mathrm{CI}$ : 0.0076, 0.1783) (middle panel of Fig 4a). We considered signatures with inconsistency rate between 0.30 to 0.18 , equivalent to actual activity ratio from 0.5 to 1.5 , as having well-refitted results. Of the originally inferred 10312 signature activities that used 96c data, 8938 (86.7\%) were well-refitted when only $80 \mathrm{c}$ data was used. 24 of 29 signatures were considered well-refitted. 
299 For the five signatures that were poorly refitted using $80 \mathrm{c}$, four of them had high $\mathrm{T}>\mathrm{C}$ mutation rates, 300 namely SBS7d, 12, 16 and 17a (left panel of Fig 4a). The inconsistency rate was significantly correlated 301 with $\mathrm{T}>\mathrm{C}$ mutation rate of signatures (Spearman's rho $=0.54, \mathrm{p}<\mathrm{e}-10$ ). We grouped the refitted data 302 based on cancer types (right panel of Fig 4a) and discovered the majority of the above five signatures 303 with inconsistent refits were each only reported in one cancer type, except for SBS17a which was present in four cancer types. SBS6 also had a high inconsistency rate and was mostly detected in nonHodgkin lymphoma (lymph-BNHL), likely due to the higher similarity shared with SBS1 (0.77). Taken together, removing $\mathrm{T}>\mathrm{C}$ mutations had a very minor impact on refitting analysis for the majority of the cases $(86.7 \%)$, apart from the minority of cases with a high $\mathrm{T}>\mathrm{C}$ mutation rate.

In addition, SBS5 and SBS40 showed noticeable differences between 96c and 80c fits in several cancer types. With the knowledge of these two 'flat' signatures are highly similar ( 0.83 using 96c; 0.86 using $80 \mathrm{c}$ ), the model could have problems distinguishing them using either $80 \mathrm{c}$ or $96 \mathrm{c}$. Thus, we suspected that the inferred signature activity of SBS5 or SBS40 could vary individually within a sample, but the sum of the activity of the two signatures would be fairly constant. We tested our hypothesis on samples with both signatures active (Fig4b). As expected, the sum of activities converged well with the mean inconsistency rate of 0.02 (95\% CI: $0.019,0.023)$, but individual attribution for SBS5 was higher by 80c (mean inconsistent rate of $0.15 ; 95 \% \mathrm{CI}: 0.14,0.16$ ) and lower for SBS40 (mean inconsistency rate of $-0.19 ; 95 \% \mathrm{CI}:-0.21,-0.16)$, and the two individual attributions were negatively correlated (Spearman's rho=-0.69, $\mathrm{p}=6.22 \mathrm{e}-164)$.

Finally, we examined signatures where removal of the $\mathrm{T}>\mathrm{C}$ mutations was most likely to be detrimental for signature identification. We compared all possible signature pairs among 65 COSMIC V3 SBS signatures (Supplemental Fig 13). As expected, the overall similarities between any two signatures tended to increase, especially for the originally dissimilar $(<0.2)$ signatures pairs (Supplemental Fig 13a and 13b). Five signature-pairs became highly similar ( $>0.8)$ using $80 \mathrm{c}$. Three out of them are reported to be biological/non-artificial mutation processes, namely SBS3-SBS5, SBS40-SBS12 and SBS40- 
80c (Supplemental Fig 13c). Therefore, we concluded that reducing to 80 channel signatures by removal of $\mathrm{T}>\mathrm{C}$ channels tended to have a minor effect on signature identification.

\section{Discussion}

330 In this study, we derived genome-wide mutational signatures that result from formalin exposure in FFPE 331 biospecimens and designed an algorithm, FFPEsig, to detect and remove artefactual-FFPE mutations 332 from measured mutational profiles. The accuracy of FFPEsig was demonstrated on synthetic FFPE samples. Accuracy was generally very high. We note poorer performance occurred when (a) biological mutation loads were low and (b) for samples where the true mutational profile closely resembled the FFPE-artefact signature - we note these circumstances are straightforward to identify in practice and so it is clear when FFPEsig can be safely applied. We note that the statistical machinery within FFPEsig is generalisable, and could be repurposed to correct for "mutational noise" from any source.

339 The repaired FFPE signature discovered in this study is highly similar to the aging signature SBS1 (Fig 340 1e). Both formalin-mutagenesis and the process leading to biological SBS1 are caused by deamination 341 of 5-methylcytosine $(5 \mathrm{mC})$ (SBS1 is due to spontaneous deamination in vivo whereas the FFPE 342 signature is caused by chemical deamination in vitro [5,24]). Unfortunately, this high similarity precludes the study of the activity of the aging signature in repaired FFPEs, which is active in all tumour genomes [24]. Similarly, the signature associated with unrepaired FFPE samples is highly similar to

345 SBS30 and therefore would also distort the study of SBS30 in FFPE samples (Fig 1d). However, 346 biological SBS30 occurs more rarely: it is caused by loss-of-function in glycosylases in BER due to 347 biallelic inactivation mutations in NTHL1, and patients carrying this variant are diagnosed as NTHL1 348 tumour syndrome with an increased lifetime risk for CRC, breast cancer, and colorectal polyposis $349[22,23,27]$. More generally, our results show that there is not necessarily a direct 1-to-1 mapping relationship from mutational process to a unique signature profile (as also questioned in [28]) as distinct mutational sources can cause similar profiles. Nevertheless, our findings speak to the utility of constructing a common carcinogen signature database $[28,29]$. 
354 The accumulation speed of $\mathrm{C}>\mathrm{T}$ artefacts in unrepaired FFPEs suggests that UDG "repair treatment" rectified DNA deamination damages to a large extent (Fig 1a). Therefore, fixation time is an important pre-analytical factor of determining the burden of FFPE-artefact mutations, which could influence the downstream signature analysis. Further, large numbers of putatively artefactual $\mathrm{T}>\mathrm{C}$ mutations can be present in FFPE samples and biological interpretation of these must be performed with extreme care. Indeed, Marchetti et al. identified 22 out of 24 (92\%) previously reported 'novel' mutations in EGFR to be FFPE artefacts, and those 22 mutations were either $\mathrm{C}>\mathrm{T}$ or $\mathrm{T}>\mathrm{C}$ [30]. So far, we have not found evidence showing which chemical agent in formalin could cause deamination of adenine, as this would result in hypoxanthine residues and further preferentially pair with cytosine to generate A:T $>\mathrm{G}: \mathrm{C}$ artefacts [31]. However, regardless of the unclear mutagenic mechanism, once the wrong residuals were generated on the DNA, multiple PCR amplifications of very small amounts of DNA from paraffinembedded tissues would make the artefacts easily observed from the data [30].

\section{Conclusion}

367 In conclusion, here we identified two mutational signatures, linked to repaired and unrepaired FFPE,

368 which are highly similar to COSMIC signatures SBS1 and SBS30, respectively. We further developed 369 FFPEsig software to accurately remove FFPE-induced mutational artefacts and demonstrated efficacy 370 in silico and in new samples. Careful application of our approach will enable the robust study of 371 mutational signatures in the enormous FFPE archives that exist around the world.

\section{Methods \& Materials}

\section{Targeted sequencing data}

374 We used targeted sequencing data from two previous publications [8,11]. Prentice et al. has collected 375 three groups of samples from CRC patients, namely fixation, baseline and blockage, to examine the impact of three factors on somatic mutation detection in clinical FFPE samples [11]. The three factors 
377

378

379

380

381

382

383

384

385

386

387

388

389

390

391

392

393

394

395

396

397

were formalin fixation time (fixation; $n=3$ ), DNA extraction kits (baseline; $n=20$ ) and storage time (blockage; $\mathrm{n}=9$ ). Samples collected in the fixation group were fixed in formalin for 2, 15, 24 and 48 hours for both repaired and unrepaired FFPEs, and paired FF samples were also available. To validate if true somatic mutations are detectable in FFPE samples, Prentice et al. applied several filters on the mutation calling results, which could have filtered FFPE artefacts out. Thus, for our purpose of learning FFPE noise signatures, we have included all data but those passed the somatic filters.

To study possible batch effects, we also included targeted panel sequencing data from study 2 in our analysis [8]. There were four normal breast tissues collected in the study. For each of them, triplicate samples were collected, fresh frozen, repaired and unrepaired FFPE. We summarised the general sample information here and more details can be found in original studies.

\section{Mutational opportunities for targeted sequencing data}

The FASTA sequences for targeted regions for studyl were downloaded from https://www.ncbi.nlm.nih.gov/sites/batchentrez and for study2 were from https://m.ensembl.org/info/website/tutorials/grch37.html. To obtain mutational opportunities, we calculated 96-channel mutation context frequency from the second to the last second nucleotide within each sequence. We assumed one genomic location was the mutated loci and added 1 count to all mutable channels with the sequence contexts of this loci. We applied this calculation over all sequences and normalised the 96-trinucleotide counts to sum up to 1 as the mutational opportunity vector for the given targeted regions (Supplemental Fig 4a and $4 \mathrm{~b}$ ). The whole genome mutation opportunity was taken from [32] (Supplemental Fig 4c).

\section{Discovery of FFPE signatures}

To derive FFPE signatures, we pre-processed the whole mutations list to exclude non-FFPE artefacts as much as possible. In both studies, mutations were excluded if they met any of the following criteria, 1) being detected in a matched FF sample; 2) being detected in matched normal samples; 3) with $>0.9$ posterior probability of being somatic mutations. The remaining mutations were used to generate 96- 
channel mutation counts by SigProfilerMatrixGenerator [33]. We normalised mutation counts from the

404 two studies separately using their corresponding mutational opportunities. Specifically, the original 405 mutation counts were divided by the mutational opportunity of the targeted regions and multiplied by mutational opportunity of whole genome context. The final normalised mutational probabilities were merged from two studies and non-T>C channels were further taken to derive FFPE signatures (Fig 1b), whereas $\mathrm{T}>\mathrm{C}$ channels were analysed separately (Supplemental Fig3c).

409

410 To derive FFPE signatures, we first applied t-distributed Stochastic Neighbour Embedding (t-SNE) for 411 dimensionality reduction for the cosine distance matrix of the merged 80-channel mutational 412 probabilities. Based on the two principal components provided by t-SNE, we defined well representative 413 samples for two repaired and unrepaired FFPE clusters using data point density estimated by gaussian 414 kernel (from scipy.stats) (Supplemental Fig 5a). The high-density samples $(>0.018)$ were used to generate one set of FFPE signature candidates. With repeating the above procedure for 100 times, we took the averaged values of each channel as the final FFPE signatures (Supplemental Fig $5 \mathrm{~b}$ and $5 \mathrm{c}$ ).

\section{Algorithm/FFPEsig for FFPE artefacts correction}

418 We denote the observed mutation counts from the FFPE sample by $V$, which was considered as a linear 419 combination of artefact signature $W_{1}$ and biological mutation frequency $W_{2}$ with their corresponding attributions/activities $H_{1}$ and $H_{2}$. Thus, we have:

$$
\boldsymbol{V} \approx \sum_{\boldsymbol{i} \in(1,2)} \boldsymbol{W}_{\boldsymbol{i}} * H_{i}
$$

In this model, $V$ and $W_{1}$ were known and the task was to infer $H=\left[H_{1}, H_{2}\right]^{T}$ and $W_{2}$. Here, we utilised

423 generalized Kullback-Leibler (KL) divergence between reconstructed $\widehat{V}=\sum_{\boldsymbol{i} \in(\mathbf{1}, \mathbf{2})} \boldsymbol{W}_{\boldsymbol{i}} * H_{i}$ and the 424 observed profile $V$ as the cost function and applied Lee and Seung's multiplicative update rules [34] to 425 minimize the cost function. 
The whole process of one iteration started with randomly generated initial values for $W_{2}$. We then updated $H$ using the multiplicative rules [34] followed by $W$, in which only $W_{2}$ was updated. From the updated $W$ and $H$, we got $\widehat{V}$. The generalised KL divergence, between $V$ and $\widehat{V}$ was computed and saved. This update process iterated over 200 steps by default until it met our termination criteria defined

431 here. We calculated the convergence ratio using the average KL divergence from the last batch of 20 iterations divided by the second last batch of 20 iterations. The algorithm would terminate if the convergence ratio reaches 0.95 . The maximum iteration by default was up to 3000 . The above one whole process provided inferred $W_{2}$ and $H$ as one candidate solution. We collected 100 candidate solutions using different random seeds and averaged them as our final solution for all samples analysed for FFPE noise correction in this study.

\section{Simulation of FFPE samples}

438 To simulated FFPE samples for algorithm performance validation, we added different amounts of FFPE artificial mutations with Poisson noise to biological mutation catalogues of 2,780 canner genomes provided in Pan-Cancer Analysis of Whole Genomes (PCAWG) project by International Cancer Genome Consortium (ICGC) [19,25]. The data is available to download from https://www.synapse.org/\#!Synapse:syn11801889. Additionally, subtype labels of PCAWG CRC samples used in the case study were also downloaded from the same site.

\section{DNA extraction and genome sequence of FFPE CRCs}

445 The male patient with ulcerative colitis was diagnosed with cancer in the transverse colon at age 48 in St. Mark’s Hospital, London, United Kingdom. Formalin-fixed paraffin-embedded (FFPE) sections of

$44710 \mu \mathrm{m}$ thickness were deparaffinized, rehydrated and lightly stained with methyl green. The annotated 448 H\&E was used as a guide for epithelial enrichment through targeted needle scraping of slides (for 449 estimated epithelial cellularity $>50 \%$ ). To collect matched normal tissue, targeted scraping of serosal 450 tissue from FFPE blocks was taken from a small intestinal segment distal to the cancer. DNA was 451 extracted using a modified protocol of the High Pure FFPE DNA Isolation Kit (Roche Life Science, 
452 Penzburg, Germany). The normal tissue DNA sample and one tumour DNA sample were repaired using 453 the NEBNext FFPE DNA Repair Mix (New England Biolabs, Inc) following the manufacturer's

454 recommendations. The remaining tumour DNA was left unrepaired. DNA libraries were prepared using 455 the NEBNext Ultra II DNA Library Prep Kit for Illumina (New England BioLabs, Ipswich, 456 Massachusetts, USA), followed by equimolar pooling strategy. Finally, all DNA libraries were 457 sequenced on NovaSeq S2 for 50bp paired end reads.

\section{Somatic variants calling in WGS FFPE CRCs}

459 The paired-end reads underwent initial quality control with FastQC [35] followed by default adaptor 460 trimming with Skewer [36] and were subsequently aligned to GRCh38 reference genome with BWA461 MEM [37]. Aligned reads were sorted by genome coordinate (SortSam, Picard) and duplicate reads were flagged with GATK's MarkDuplicates [38]. The two FFPE tumour samples were called against the matched normal separately using the Mutect2 somatic variant caller from GATK [38]. Variants were marked with filters by FilterMutectCalls. Variants were kept if they were PASS by Mutect2, aligned to a canonical chromosome, had a total allelic depth of greater or equal to 10 in both the tumour and normal sample and had 3 or more reads supporting the alternative allele in the tumour sample. The filtered variants from two FFPE tumour samples were merged into a single VCF file using VCFtools [39].

We used Platypus on the merged VCF file as the candidate somatic variant list and integrated local alignment with multi-sample variant calling to assess the evidence for these variants across all samples [40]. The resulting VCF file was further filtered to only contain variants 1) if the FILTER flag was

472 PASS or other acceptable filters (alleleBias, Q20, QD, SC, HapScore); 2) the variant was not a known 473 germline variant; 3 ) a genotype was called for all samples; the genotype phred score was 10 or more in 474 all samples; 4) the normal sample had no reads containing the variant and at least 3 or more reads supported the variant in a tumour sample. Variants present in two FFPE samples with 5 or more supporting reads were classified as concordant mutations. 


\section{$477 \quad$ Signature refitting analysis}

478 To validate if signature refitting analysis could use 80-channel spectra without $\mathrm{T}>\mathrm{C}$, we dropped $\mathrm{T}>\mathrm{C}$

479 mutation channels of COSMIC SBS signatures and renormalised them to sum up to 1 . The original

480 activities inferred using 96-channel signatures for PCAWG cohorts were obtained from

481 https://dcc.icgc.org/releases/PCAWG/mutational_signatures/ $[19,25]$. The active signatures for each

482 sample were selected if the original activities $>0$. We next refitted $80 \mathrm{c}$ and $96 \mathrm{c}$ active signatures to the

483 mutational catalogues with and without $\mathrm{T}>\mathrm{C}$ mutations accordingly using our locally implemented

484 refitting algorithm to exclude possible bias introduced by different tools. The refitting algorithm used the same multiplicative update rules and termination criteria from FFPEsig, but was different in two aspects, 1) the number of signatures was flexible which depended on the active signatures in each sample; 2) only $H$ was updated in each iteration. The inferred activities for 80c-signatures were then rescaled by dividing total mutation frequencies of non- $\mathrm{T}>\mathrm{C}$ mutation channels of $96 \mathrm{c}$ spectra. The rescaled $80 \mathrm{c}$ attributions were used to compare to those inferred from $96 \mathrm{c}$ signatures.

\section{Data and code access}

491 Submission of BAM files of sequenced data to EGA is in progress. The VCF files generated in our 492 study are available from the corresponding authors, upon reasonable request. FFPEsig is implemented 493 in python which is available to download from https://github.com/QingliGuo/FFPEsig, as well as 494 analysis code and data used in this study.

\section{Abbreviations}

496 FFPE: Formalin fixation and paraffin embedding

497 FF: fresh frozen

498 UDG: uracil DNA glycosylase

499 PCAWG: Pan-Cancer Analysis of Whole Genomes

500 COSMIC: The Catalogue of Somatic Mutations in Cancer

501 SBS: single base substitutions 
502 CRC: colorectal cancer

503 MSI: microsatellite instability

504 POLE: proofreading subunit of polymerase epsilon

505 MSS: microsatellite stability

506 EGFR: epidermal growth factor receptor

507 PCR: polymerase chain reaction

508 BAM: Binary Alignment Map file

509 t-SNE: t-distributed Stochastic Neighbour Embedding

510 Acknowledgements

511 We thank Virinder Singh Reen and Dr. Ignacio Vázquez-García for helpful comments on the 512 manuscript. We thank the team at St Mark's Hospital London, UK, Andrea Sottoriva, Inma Spiteri,

513 Ann-Marie Baker, Salpie Nowinski, Jacob Househam and Chris Kimberley for support with FFPE 514 sample provision and analysis. This research utilised Queen Mary's Apocrita HPC facility, supported

515 by QMUL Research-IT. http://doi.org/10.5281/zenodo.438045. We also thank Prof. Amy C. Degnim,

516 Dr. Chen Wang and the Mayo Clinic for help with data sharing.

\section{Author's contributions}

518 Q.G, T.A.G and V.M. conceived the study. Q.G. designed, carried out the data analysis, designed and 519 implemented the algorithm and interpreted the initial results. V.M. designed the algorithm and 520 supervised data analysis. Q.G and E.L. carried out the WGS FFPE case study. I.AB provided FFPE 521 samples and performed genome sequencing. K.C performed mutation calling on the FFPE case. Q.G., 522 V.M., T.A.G. and E.L. participated and contributed in results discussion and interpretation. Q.G. and T.A.G wrote the manuscript. E.L. and V.M edited the manuscript. V.M. and T.A.G supervised the project. All authors read and approved the final manuscript. 


\section{Funding}

526 T.A.G acknowledges funding from Cancer Research UK (A19771 and A16581) and the Barts Charity

527 (472-2300). E.L. is also supported by funding from Cancer Research UK (A19771).

\section{Ethics approval and consent to participate}

529 The archival colorectal cancer studied was collected and analysed in accordance with ethical approval 530 from the UK Research Ethics Committee (REC: 18/LO/2051 IRAS:249008 - Fulham committee). The 531 sample was anonymised to the researchers.

\section{Competing interests}

533 All authors named in this paper declare no conflicts of interest.

\section{References}

1. Williams C, Pontén F, Moberg C, Söderkvist P, Uhlén M, Pontén J, et al. A high frequency of sequence alterations is due to formalin fixation of archival specimens. Am J Pathol. 1999;155:146771.

2. Mathieson W, Thomas G. Using FFPE Tissue in Genomic Analyses: Advantages, Disadvantages and the Role of Biospecimen Science. Curr Pathobiol Rep. Current Pathobiology Reports; 2019;7:35-40.

3. Spencer DH, Sehn JK, Abel HJ, Watson MA, Pfeifer JD, Duncavage EJ. Comparison of clinical targeted next-generation sequence data from formalin-fixed and fresh-frozen tissue specimens. J Mol Diagn. American Society for Investigative Pathology; 2013;15:623-33.

4. Wong SQ, Li J, Tan AYC, Vedururu R, Pang JMB, Do H, et al. Sequence artefacts in a prospective series of formalin-fixed tumours tested for mutations in hotspot regions by massively parallel sequencing. BMC Med Genomics [Internet]. 2014;7. Available from: http://dx.doi.org/10.1186/17558794-7-23

5. Do H, Dobrovic A. Sequence artifacts in DNA from formalin-fixed tissues: Causes and strategies for minimization. Clin Chem. 2015;61:64-71.

6. Kawanishi M, Matsuda T, Yagi T. Genotoxicity of formaldehyde: molecular basis of DNA damage and mutation. Front Environ Sci Eng China. 2014;2:36.

7. Kennedy-Darling J, Smith LM. Measuring the formaldehyde protein-DNA cross-link reversal rate. Anal Chem. 2014;86:5678-81.

8. Bhagwate AV, Liu Y, Winham SJ, McDonough SJ, Stallings-Mann ML, Heinzen EP, et al. Bioinformatics and DNA-extraction strategies to reliably detect genetic variants from FFPE breast tissue samples. BMC Genomics. BMC Genomics; 2019;20:1-10. 
9. Arbeithuber B, Makova KD, Tiemann-Boege I. Artifactual mutations resulting from DNA lesions limit detection levels in ultrasensitive sequencing applications. DNA Res. 2016;23:547-59.

10. Chen G, Mosier S, Gocke CD, Lin MT, Eshleman JR. Cytosine Deamination Is a Major Cause of Baseline Noise in Next-Generation Sequencing. Mol Diagn Ther. 2014;18:587-93.

11. Prentice LM, Miller RR, Knaggs J, Mazloomian A, Hernandez RA, Franchini P, et al. Formalin fixation increases deamination mutation signature but should not lead to false positive mutations in clinical practice. PLoS One. 2018;13:e0196434.

12. Nik-Zainal S, Alexandrov LB, Wedge DC, Van Loo P, Greenman CD, Raine K, et al. Mutational processes molding the genomes of 21 breast cancers. Cell. 2012;149:979-93.

13. Alexandrov LB, Nik-Zainal S, Wedge DC, Aparicio SAJR, Behjati S, Biankin AV, et al. Signatures of mutational processes in human cancer. Nature. 2013;500:415-21.

14. Pich O, Muiños F, Lolkema MP, Steeghs N, Gonzalez-Perez A, Lopez-Bigas N. The mutational footprints of cancer therapies. Nat Genet. Springer US; 2019;51:1732-40.

15. Ma J, Setton J, Lee NY, Riaz N, Powell SN. The therapeutic significance of mutational signatures from DNA repair deficiency in cancer. Nat Commun. Springer US; 2018;9:1-12.

16. Gulhan DC, Lee JJK, Melloni GEM, Cortés-Ciriano I, Park PJ. Detecting the mutational signature of homologous recombination deficiency in clinical samples. Nat Genet. Springer US; 2019;51:912-9.

17. Van Hoeck A, Tjoonk NH, Van Boxtel R, Cuppen E. Portrait of a cancer: Mutational signature analyses for cancer diagnostics. BMC Cancer. BMC Cancer; 2019;19:1-14.

18. Donoghue MTA, Schram AM, Hyman DM, Taylor BS. Discovery through clinical sequencing in oncology. Nature Cancer. Nature Publishing Group; 2020;1:774-83.

19. Alexandrov LB, Kim J, Haradhvala NJ, Huang MN, Tian Ng AW, Wu Y, et al. The repertoire of mutational signatures in human cancer. Nature. 2020;578:94-101.

20. Maaten L, Hinton G. Visualizing data using t-SNE. J Mach Learn Res [Internet]. jmlr.org; 2008; Available from: http://www.jmlr.org/papers/v9/vandermaaten08a.html

21. Kim S, Park C, Ji Y, Kim DG, Bae H, van Vrancken M, et al. Deamination Effects in FormalinFixed, Paraffin-Embedded Tissue Samples in the Era of Precision Medicine. J Mol Diagn. American Society for Investigative Pathology and the Association for Molecular Pathology; 2017;19:137-46.

22. Drost J, Van Boxtel R, Blokzijl F, Mizutani T, Sasaki N, Sasselli V, et al. Use of CRISPR-modified human stem cell organoids to study the origin of mutational signatures in cancer. Science. 2017;358:234-8.

23. Grolleman JE, de Voer RM, Elsayed FA, Nielsen M, Weren RDA, Palles C, et al. Mutational Signature Analysis Reveals NTHL1 Deficiency to Cause a Multi-tumor Phenotype. Cancer Cell. 2019;35:256-66.e5.

24. Alexandrov LB, Jones PH, Wedge DC, Sale JE, Campbell PJ, Nik-Zainal S, et al. Clock-like mutational processes in human somatic cells. Nat Genet. Nature Publishing Group; 2015;47:1402-7.

25. ICGC/TCGA Pan-Cancer Analysis of Whole Genomes Consortium. Pan-cancer analysis of whole genomes. Nature. 2020;578:82-93.

26. Niu B, Ye K, Zhang Q, Lu C, Xie M, Mclellan MD, et al. BIOINFORMATICS APPLICATIONS NOTE Sequence analysis MSIsensor : microsatellite instability detection using paired tumor-normal 
sequence data. 2014;30:1015-6.

27. Kuiper RP, Nielsen M, De Voer RM, Hoogerbrugge N. NTHL1 Tumor Syndrome. In: Adam MP, Ardinger HH, Pagon RA, Wallace SE, Bean LJH, Mirzaa G, et al., editors. GeneReviews ${ }^{\circledR}$. Seattle (WA): University of Washington, Seattle; 2020.

28. Koh G, Zou X, Nik-Zainal S. Mutational signatures: Experimental design and analytical framework. Genome Biol. Genome Biology; 2020;21:1-13.

29. Kucab JE, Zou X, Morganella S, Joel M, Nanda AS, Nagy E, et al. A Compendium of Mutational Signatures of Environmental Agents. Cell. Elsevier Inc.; 2019;177:821-36.e16.

30. Marchetti A, Felicioni L, Buttitta F. Assessing EGFR mutations. N. Engl. J. Med. 2006. p. 526-8; author reply 526-8.

31. Karran P, Lindahl T. Hypoxanthine in deoxyribonucleic acid: generation by heat-induced hydrolysis of adenine residues and release in free form by a deoxyribonucleic acid glycosylase from calf thymus. Biochemistry. 1980;19:6005-11.

32. Fischer A, Illingworth CJR, Campbell PJ, Mustonen V. EMu: Probabilistic inference of mutational processes and their localization in the cancer genome. Genome Biol. 2013;14:R39.

33. Bergstrom EN, Huang MN, Mahto U, Barnes M, Stratton MR, Rozen SG, et al. SigProfilerMatrixGenerator: A tool for visualizing and exploring patterns of small mutational events. BMC Genomics. 2019;20:685.

34. Lee DD, Sebastian Seung H. Learning the parts of objects by non-negative matrix factorization. Nature. 1999;401:788-91.

35. Andrews S, Krueger F, Segonds-Pichon A, Biggins L, Krueger C, Wingett S. FastQC: a quality control tool for high throughput sequence data [Internet]. 2010. Available from: http://www.bioinformatics.babraham.ac.uk/projects/fastqc

36. Jiang H, Lei R, Ding S-W, Zhu S. Skewer: a fast and accurate adapter trimmer for next-generation sequencing paired-end reads. BMC Bioinformatics. 2014;15:182.

37. Li H, Durbin R. Fast and accurate short read alignment with Burrows-Wheeler transform. Bioinformatics. Oxford Academic; 2009;25:1754-60.

38. Van der Auwera GA, Carneiro MO, Hartl C, Poplin R, Del Angel G, Levy-Moonshine A, et al. From FastQ data to high confidence variant calls: the Genome Analysis Toolkit best practices pipeline. Curr Protoc Bioinformatics. 2013;43:11.10.1-11.10.33.

39. Danecek P, Auton A, Abecasis G, Albers CA, Banks E, DePristo MA, et al. The variant call format and VCFtools. Bioinformatics. 2011;27:2156-8.

40. Rimmer A, Phan H, Mathieson I, Iqbal Z, Twigg SRF, WGS500 Consortium, et al. Integrating mapping-, assembly- and haplotype-based approaches for calling variants in clinical sequencing applications. Nat Genet. 2014;46:912-8. 
a

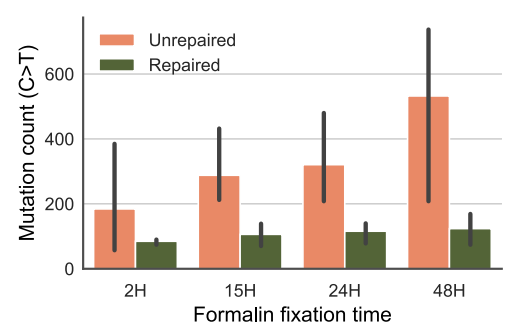

b

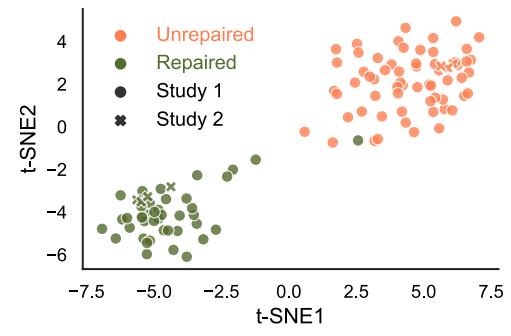

d

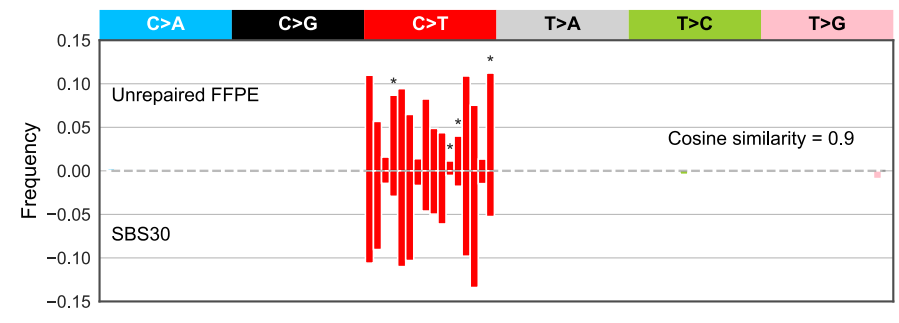

e

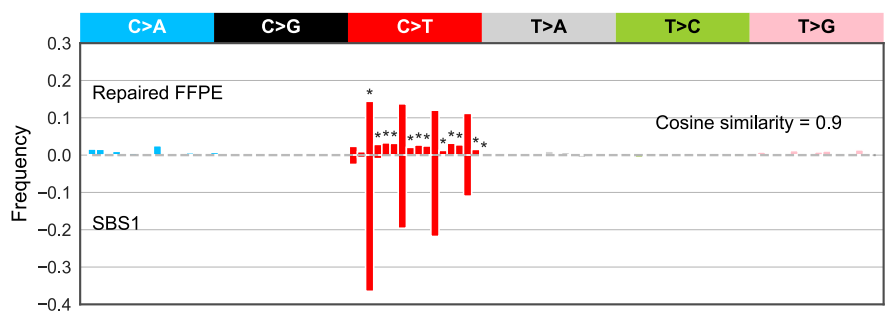

C

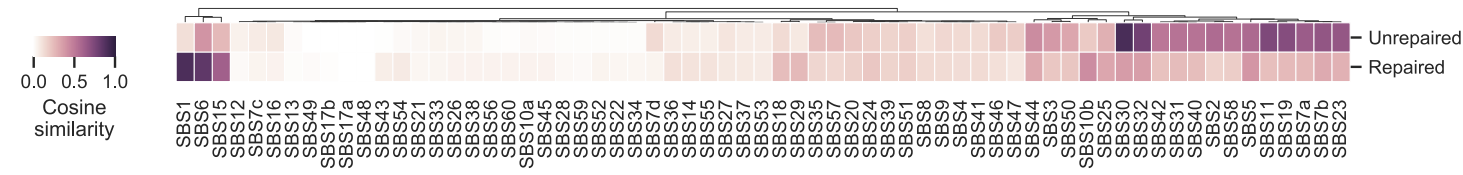

Fig1. FFPE artefact signatures. (a) $\mathrm{C}>\mathrm{T}$ mutation count in FFPE samples increases with formalin fixation time. We used FFPE-only $\mathrm{C}>\mathrm{T}$ mutations, referring to $\mathrm{C}>\mathrm{T}$ mutations that are only discovered in FFPE samples not in matched FF. The error bar shows standard deviation for measurements made on three individuals. (b) Cluster of $n=110$ normalised FFPE mutational profiles from two different studies [11,8]. The cluster is represented by t-SNE on cosine metric of normalised 80-channel (without T $>$ C) FFPE-only mutational profiles (see Methods \& Materials). Each FFPE sample is classified as unrepaired (with UDG treatment; pink dots) or repaired (without UDG; green dots). The two studies are marked using circle or cross shape. (c) Comparison of FFPE signatures to COSMIC V3 SBS signatures. (d) Unrepaired FFPE signature is highly similar to SBS30. C $>\mathrm{T}$ mutation channels with fold change over 2 are marked with asterisk. (e) Repaired FFPE signature is highly similar to SBS1. 
a

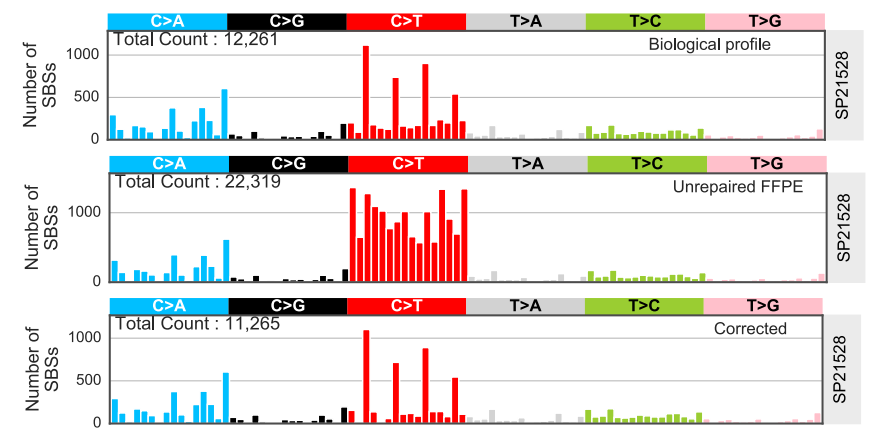

b

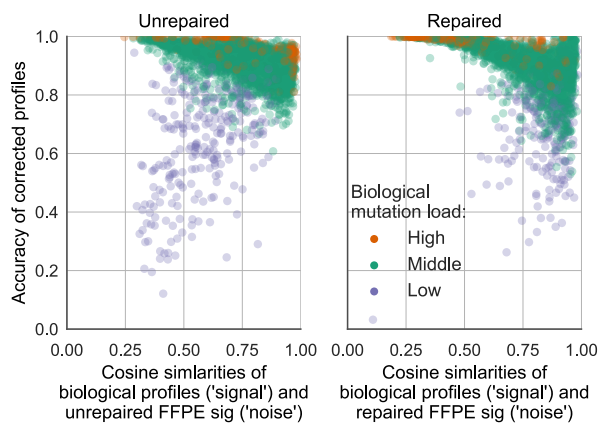

C Percentage (\%) of samples with correction accuracy above 0.90

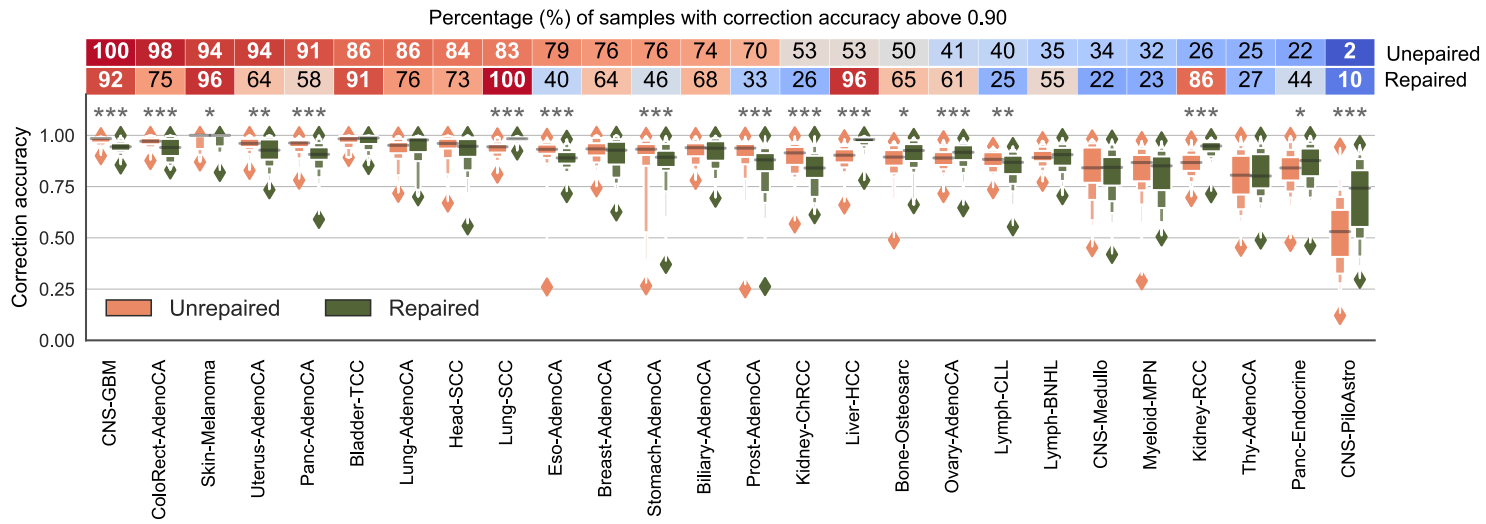

d

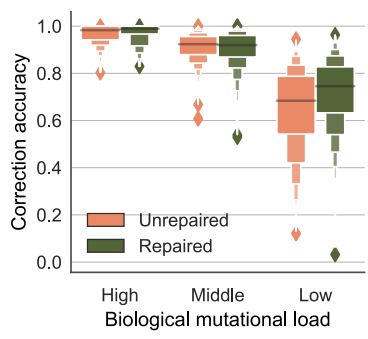

e

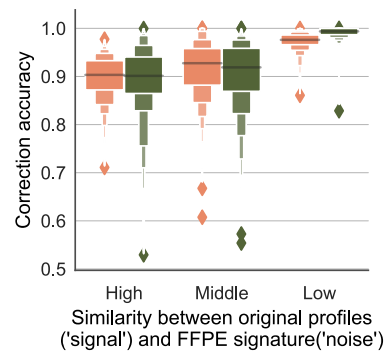

$f$

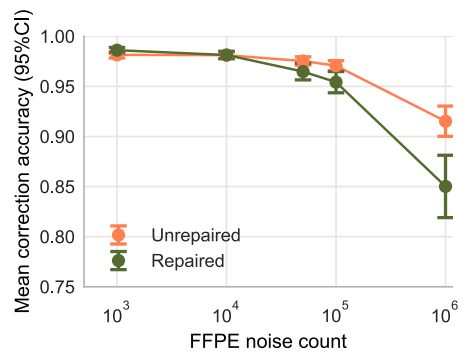

Fig2. Correction of FFPE artefacts in synthetic FFPE samples. We added FFPE noise signatures to biological mutational profiles in PCAWG dataset [20] to simulate FFPE mutational profiles. (a) Artefacts correction result of one colorectal cancer (SP21528). From top to bottom, the three panels are original/biological mutational profile, simulated FFPE mutational profile (unrepaired) and the corrected somatic mutation catalogue. (b) Correction accuracy for all simulated data. The left panel shows results for unpaired FFPEs and the right panel is for repaired FFPEs. The $\mathrm{x}$-axis shows cosine similarities between original profiles ('signal') and the FFPE signatures ('noise'). We also group the data into three categories according to the biological mutation load, namely high (top 10\%, orange dots), low (bottom $10 \%$, purple dots) and middle (the remaining ones, green dots). (c) Correction accuracy for different cancer types. Cancer types with at least 20 samples are used here. The difference between unrepaired and repaired FFPE correction accuracy is shown above each box-pair using two-sided Mann-Whitney $U$ test. $P$ value $<=0.001(* * *) ; 0.001<p$ value $<=0.01(* *) ; 0.01<p$ value $<=0.05(*) ; p$ value $>0.05$ (none). The percentages of well-corrected samples (accuracy $>0.9$ ) are annotated in the top colour bars. (d) Correction accuracy positively correlates with biological mutation load. (e) Correction accuracy negatively correlates with with similarities between 'signal' and 'noise'. The three categories are use high (top 10\%), low (bottom 10\%) and middle (the remaining ones). (f) Correction accuracy drops with increasing FFPE artefacts in both types of FFPEs. We selected cancer types with at least $80 \%$ wellcorrected samples in both unrepaired and repaired FFPEs from (c). The results are collected from simulated samples added with five different noise levels from $10^{3}, 10^{4}, 5 \times 10^{4}, 10^{5}$ to $10^{6}$. The $95 \%$ confidence interval of each mean correction accuracy is marked using error bar here. 
a
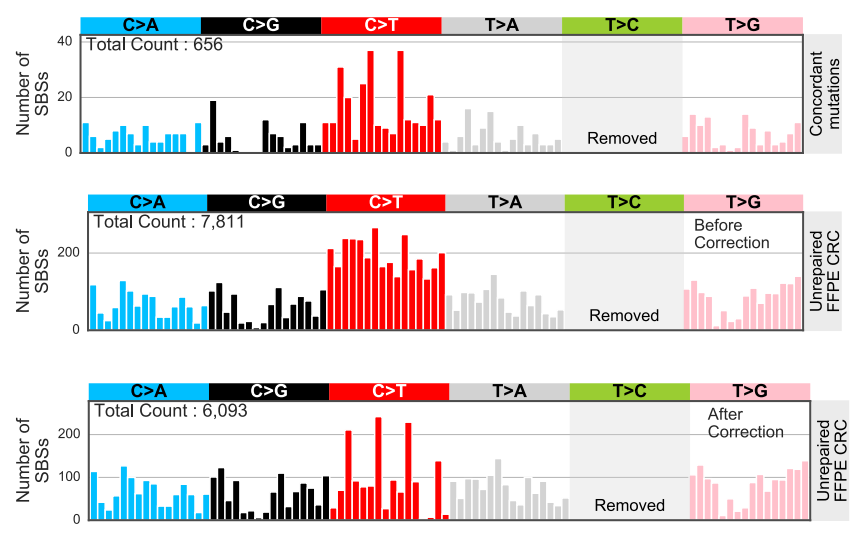

b

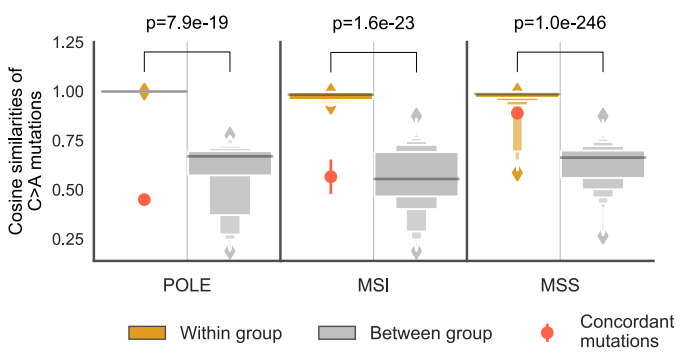

C

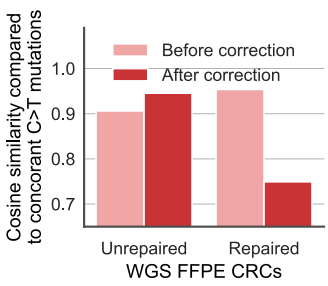

d

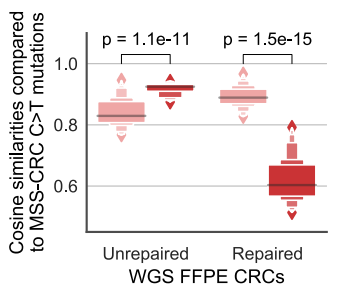

e

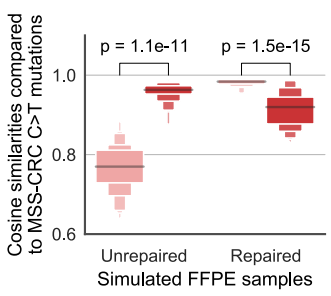

Fig3. A case study of applying FFPE artefact correction method on two WGS CRC FFPE samples. The two FFPE samples are from the same CRC patient. One of FFPEs is unrepaired and the other one is repaired. (a) Correction result for the unrepaired FFPE sample. The three panels are concordant mutation catalogues (top), unrepaired FFPE CRC profiles before correction (middle) and after correction (bottom). Concordant mutations refer to variants are shared between repaired and unrepaired FFPEs with at least 5 reads supporting the variant, and their profile is taken as an approximation of true mutational catalogue of the tumour. We removed $\mathrm{T}>\mathrm{C}$ mutations to show clear pattern of other mutation channels due to their large numbers. (b) Concordant $\mathrm{C}>\mathrm{A}$ mutation profile is highly similar to MSS-CRC C $>\mathrm{A}$ mutation patterns. PCAWG CRCs are grouped based on their known labels, namely POLE, MSI and MSS. The sample-pair cosine similarities of $\mathrm{C}>\mathrm{A}$ mutation patterns within and between subgroups are shown in orange and grey box plot, respectively. The $p$-values of differences for each subgroup are shown above each box-pair using two-sided Mann-Whitney $U$ test. The error bar shows standard deviation.(c) Comparing correction results of two FFPE samples to concordant mutations. As the correction acts on $\mathrm{C}>\mathrm{T}$ mutation channels, we compared the cosine similarity changes of original profile (pink colour) and corrected profile (red colour) on $\mathrm{C}>\mathrm{T}$ channels. (d) Comparing correction results of two FFPE samples to MSS-CRCs. (e) Comparing correction results of simulated MSS-CRC FFPE profiles. We compared each simulated MSS-CRC FFPE sample to all other MSS-CRC profiles but their real biological profile to treat them the same way as our WGS FFPE samples, for which the FF sample is not available. 
a
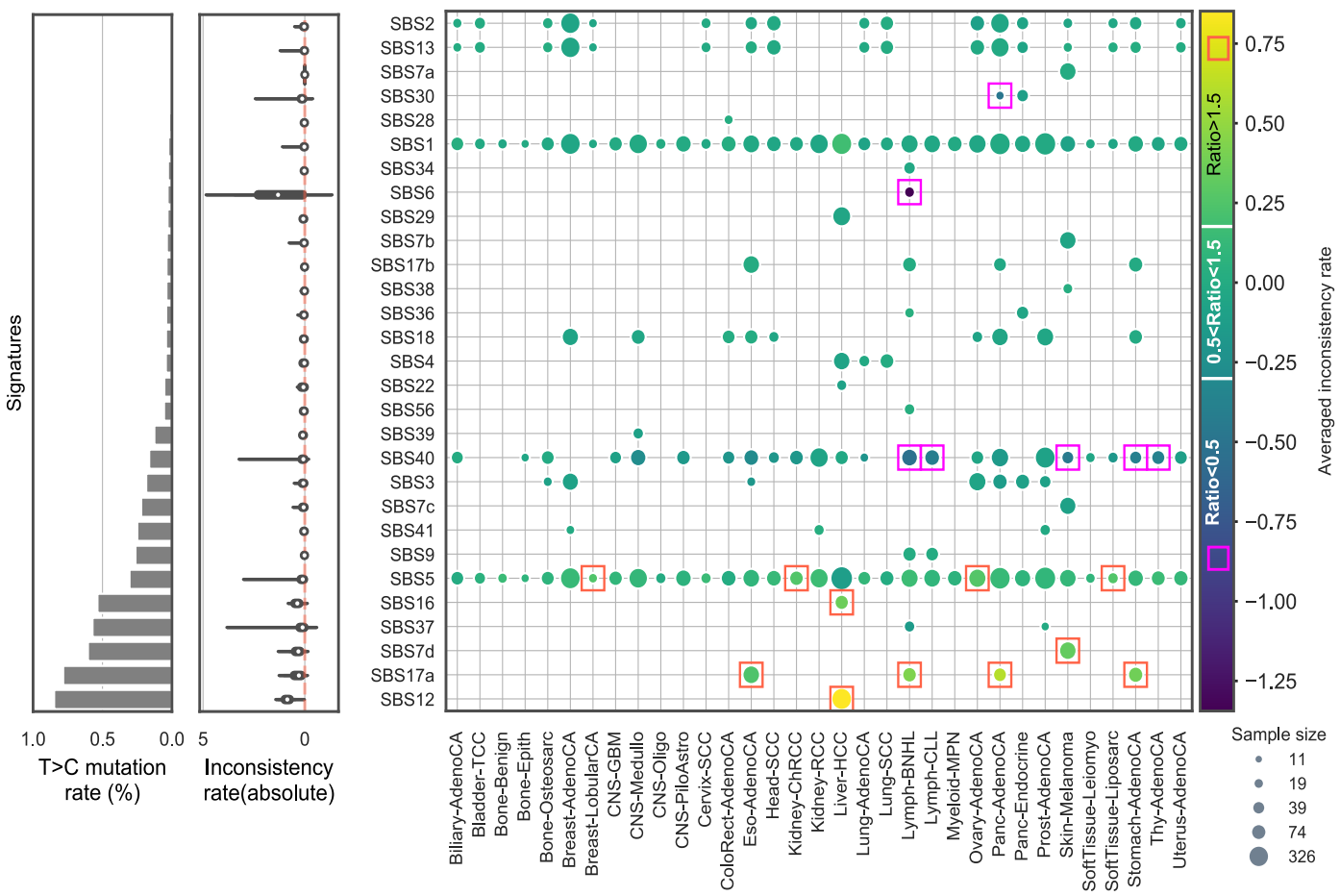

b

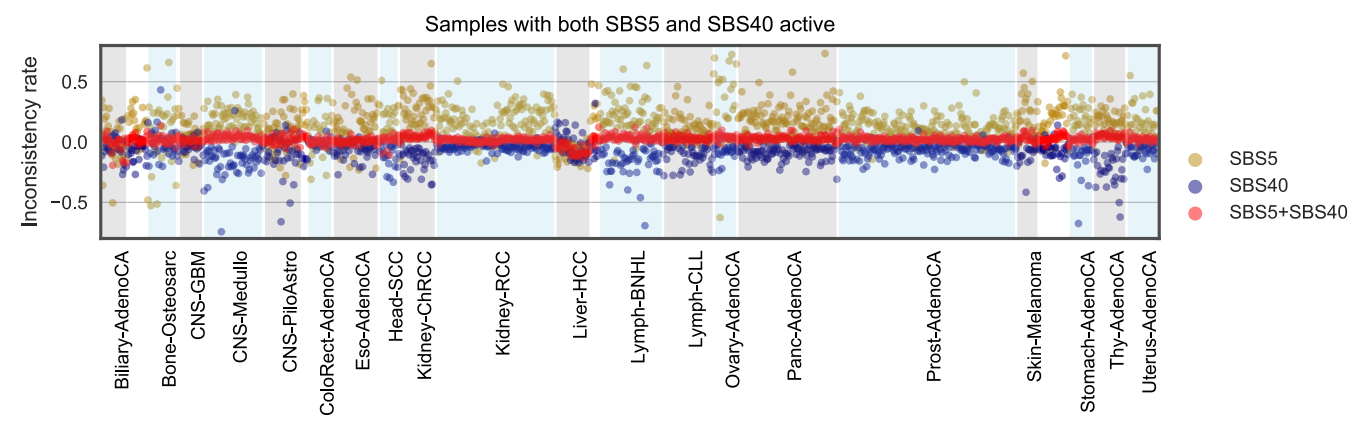

Fig4. Comparison of signature activities inferred by signatures with and without $\mathrm{T}>\mathrm{C}$ mutations. We inferred signature activities using 96-channel (96c) and 80-channel (80c; without $\mathrm{T}>\mathrm{C}$ ) signatures on PCAWG mutational profiles. Here we use inconsistency rate as a measurement for how well the inferred activities agree with each other. Inconsistency rate is calculated as $\log 10$ (activity_80c/activity_96c). (a) Activities inferred by 96c and 80c signatures are consistent for majority of signatures. Left panel: sum of mutational probabilities in $\mathrm{T}>\mathrm{C}$ channels for each signature. Middle panel: violin plot of absolute inconsistency rate for all signatures. Right panel: heatmap of mean inconsistency rate for all signatures in different cancer types. Orange rectangle marks the average activity ratio (activity_80c/activity_96c) above 1.5 ( $\sim 0.18$ on $\log 10$ scale), which means $80 \mathrm{c}$ activity is bigger than 1.5 times of $96 \mathrm{c}$ activity. The purple rectangle marks the averaged activity ratio below $0.5(\sim-0.30$ on $\log 10$ scale), which means $80 \mathrm{c}$ activity is smaller than $50 \%$ of $96 \mathrm{c}$ activity. The radius of each circle represents the sample size (in log scale). (b) Activity flows between two similar signatures (SBS5 and SBS40). The inconsistency rates for SBS5 in all samples are in golden dots, and those for SBS40 are in blue dots. The inconsistency rate for the sum activity of SBS5 and SBS40 is shown in red dots. 
Supplemental Figures 
a

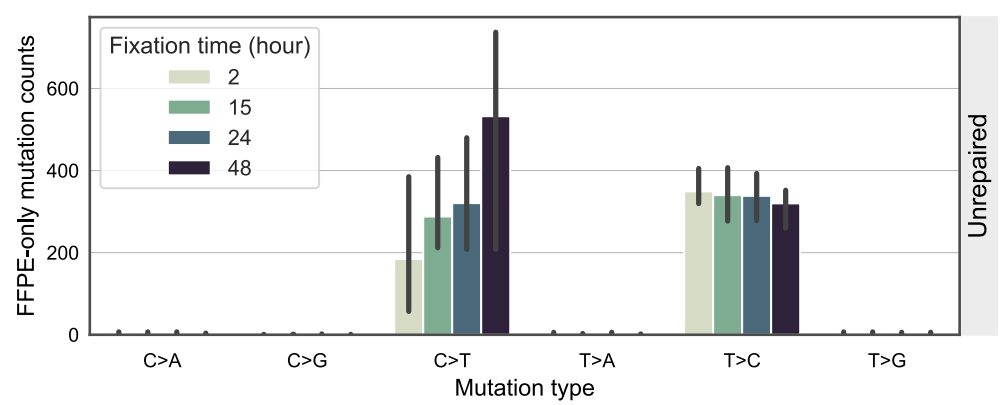

b

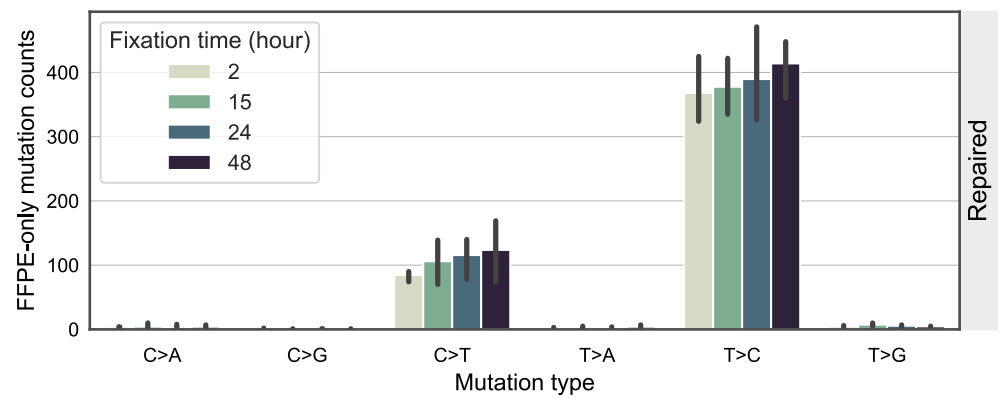

Supplemental Fig1. FFPE-only mutations with increasing formalin fixation time. FFPEonly mutations here refer to those are not present in matched FF sample and the data is from fixation group in study 1 [11] (see Methods \& Materials). (a) Mutation count for six mutation types in unrepaired FFPE samples (without UDG treatment). For each mutation type, we show the mutation counts detected in four FFPE samples being fixed in formalin for 2, 15, 24 and 48 hours respectively. All data is collected from three patients. The error bar shows standard deviation for measurements made on three individuals. (b) Mutation count in repaired FFPE samples (with UDG treatment). 
a

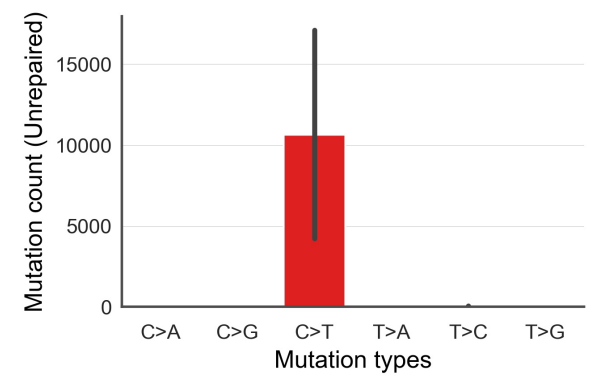

b

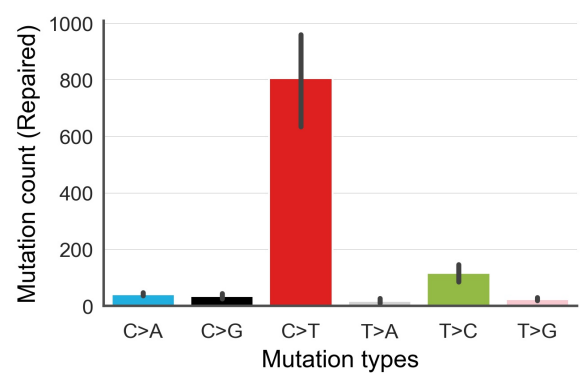

Supplemental Fig2. FFPE-only mutations in six basic mutation types in study 2. FFPE-only mutations here refer to those are not present in matched FF sample. The data is collected from four patients in study 2 [8] (see Methods \& Materials). (a) for unrepaired FFPEs. (b) for repaired FFPEs. The error bar shows standard deviation for measurements made on four individuals. 

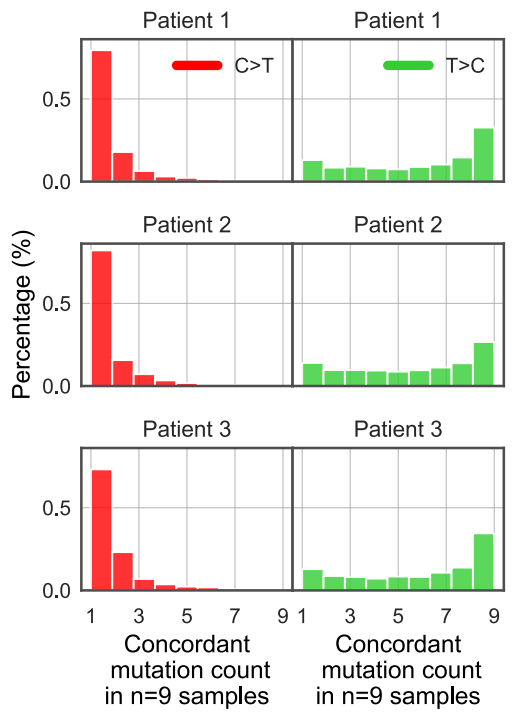

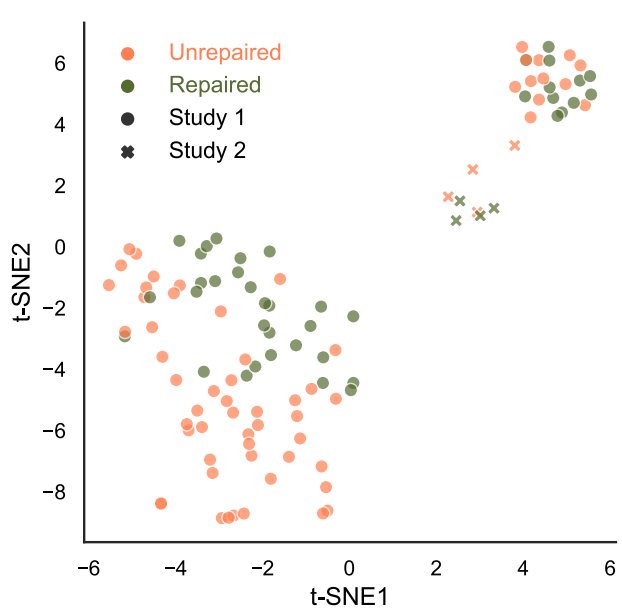

b

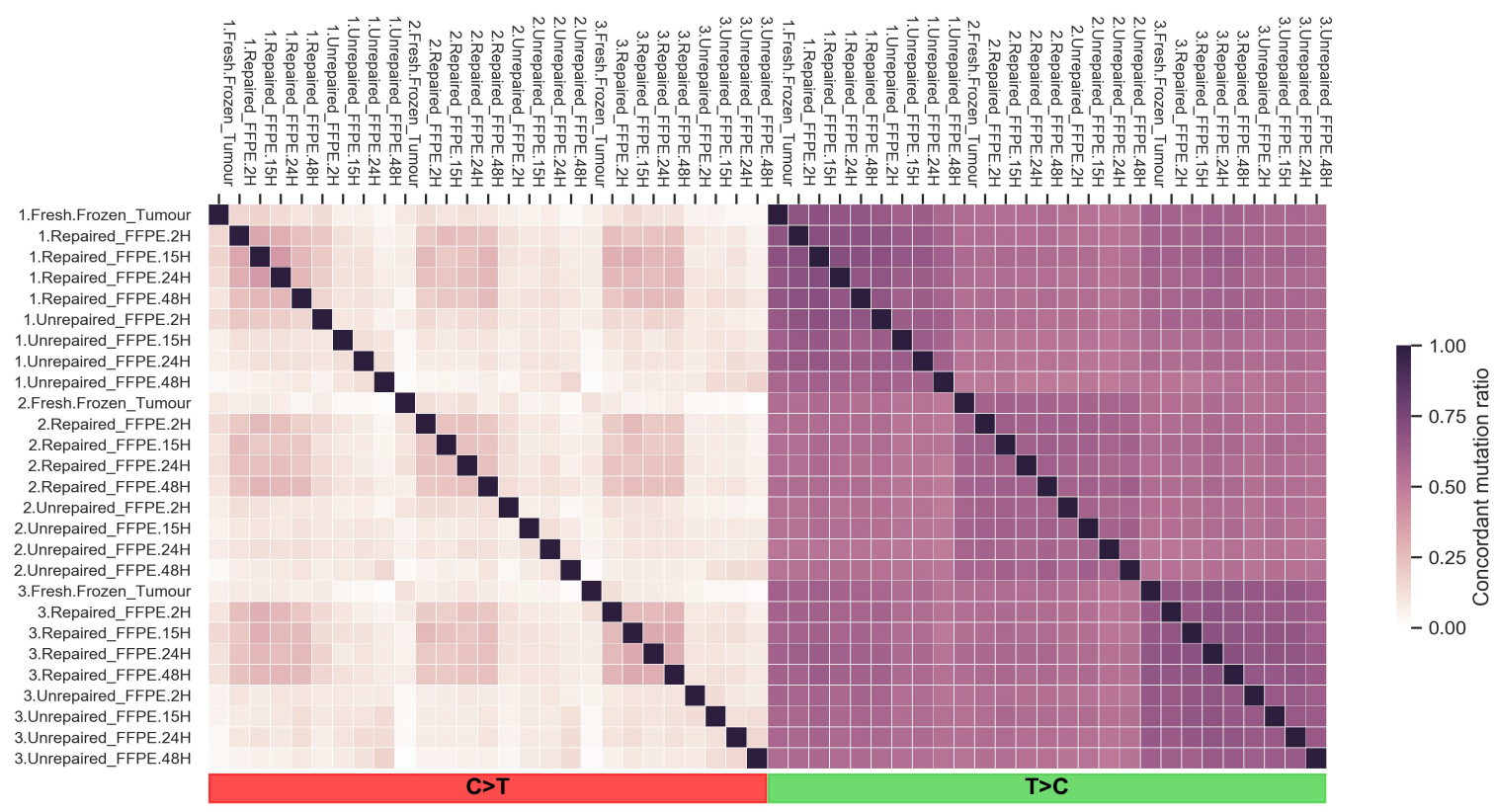

Supplemental Fig3. $\mathbf{T}>\mathrm{C}$ mutations are highly repeated among samples with no specific error profile. We use all mutation data of fixation group $(n=27)$ from study 1 for (a) and (b) as $\mathrm{T}>\mathrm{C}$ are only over-represented in study 1 . We used FFPE-only $\mathrm{T}>\mathrm{C}$ mutations of all FFPEs $(\mathrm{n}=110)$ from study 1 and 2 in (c). (a) Normalised histogram of concordant mutation count per patient. We take all $\mathrm{T}>\mathrm{C}$ and $\mathrm{C}>\mathrm{T}$ mutations from the whole mutation list and counted the occurrences for the unique set of all mutations among all samples from each patient $(n=9 ; 4$ repaired FFPE + 4 unrepaired FFPE +1 FF). (b) Pair-wise comparison of concordant mutation ratios for all samples from three patients $(n=27)$. Concordant mutation ratio is calculated using concordant mutation numbers of a sample-pair divided by unique mutation count in the sample pair. (c) Clusters of $\mathrm{T}>\mathrm{C}$ mutation profiles over 110 FFPE samples. It is the same plot as Fig $1 \mathrm{~b}$ but using 16-channel of $\mathrm{T}>\mathrm{C}$ mutation data whereas Fig $1 \mathrm{~b}$ using 80 -channel without $\mathrm{T}>\mathrm{C}$ mutations. The cluster is represented by t-SNE on cosine metric of 16-channel $\mathrm{T}>\mathrm{C}$ mutational profiles which are normalized using targeted-region mutational opportunities and whole genome mutational contexts (see Methods \& Materials). 
a

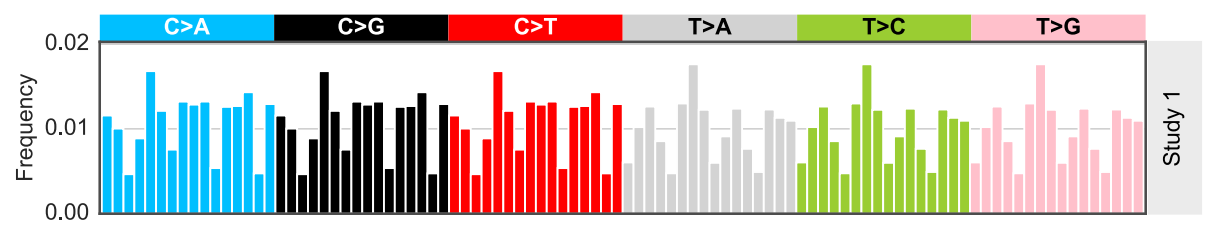

b

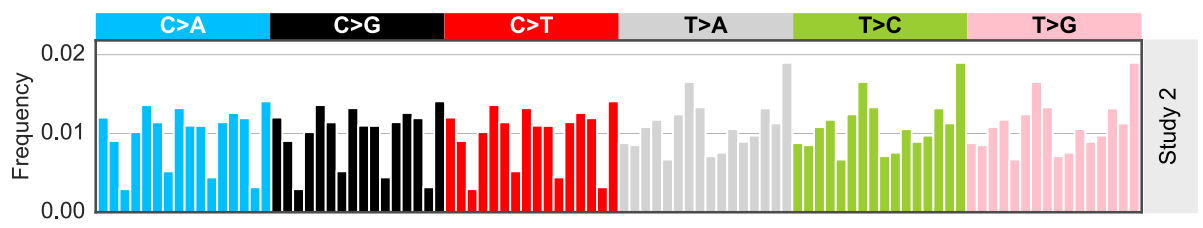

C

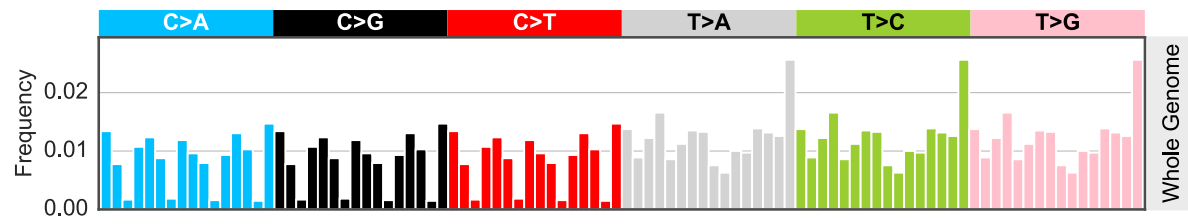

Supplemental Fig4. Mutational opportunities (a) of study 1 targeted regions (b) of study 2 targeted regions (c) of whole genome sequence context 


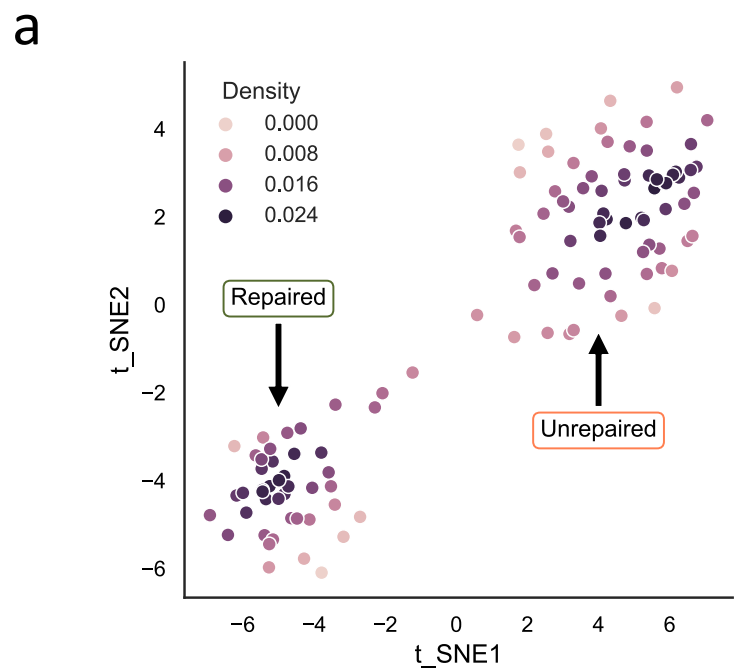

b

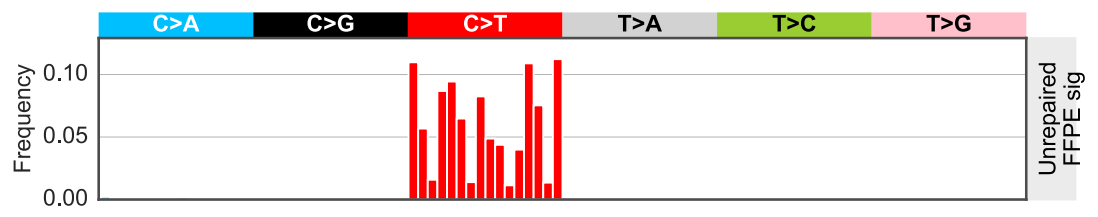

C

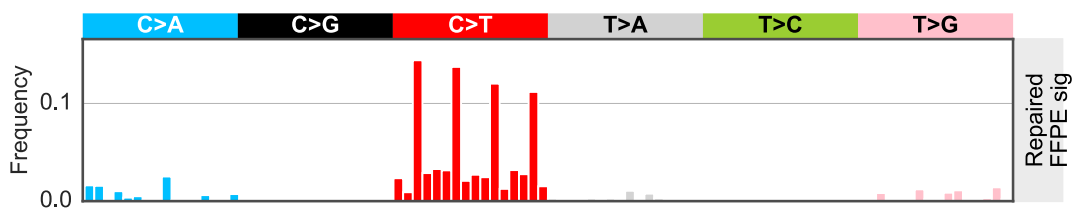

Supplemental Fig5. Deriving FFPE signatures from well-representative samples from t-SNE clustering result. (a) Scatter plot of spatial density of t-SNE clustered samples measured using gaussian kernel. The t-SNE cluster is the same as Fig $1 \mathrm{~b}$ but with spatial density instead. Samples with density value over 0.018 are classified as well-representative samples, and one FFPE signature candidate are generated by averaging the mutational channels. (b) Final version of unrepaired FFPE signature. We repeated (a) for 100 times using different random seeds, thus we have 100 unrepaired FFPE signature candidates. The final version of unrepaired FFPE signature takes the averaged values of all 100 candidates. (c) Final version of repaired FFPE signature. It is derived from the same method as used in (b). 


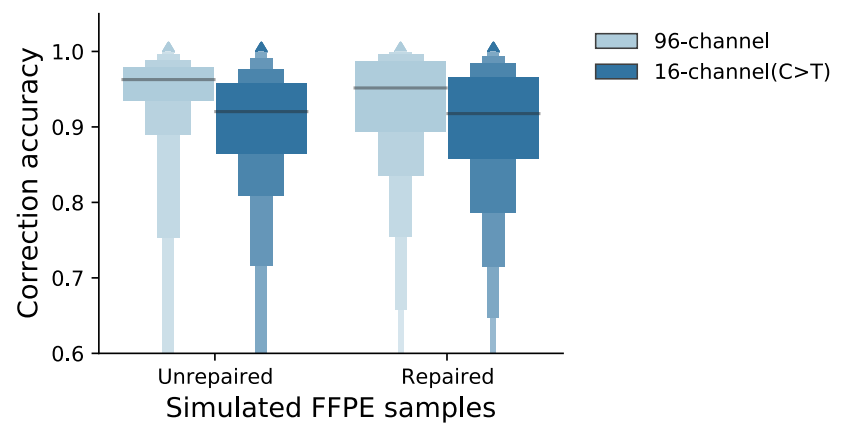

Supplemental Fig6. Comparison of correction accuracy measured using all mutations (96-channel) versus using $\mathrm{C}>\mathrm{T}$ mutations. 
a
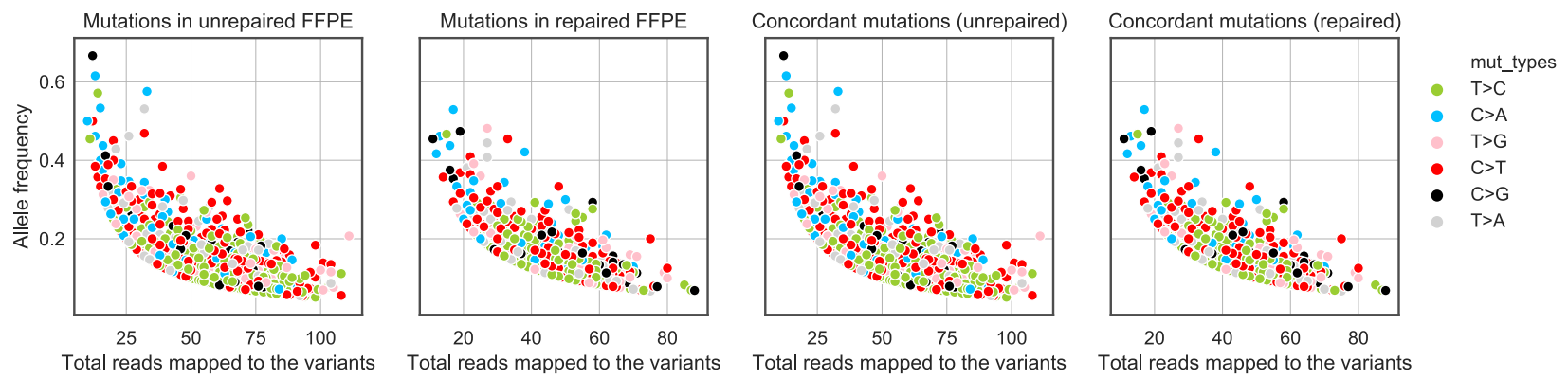

b
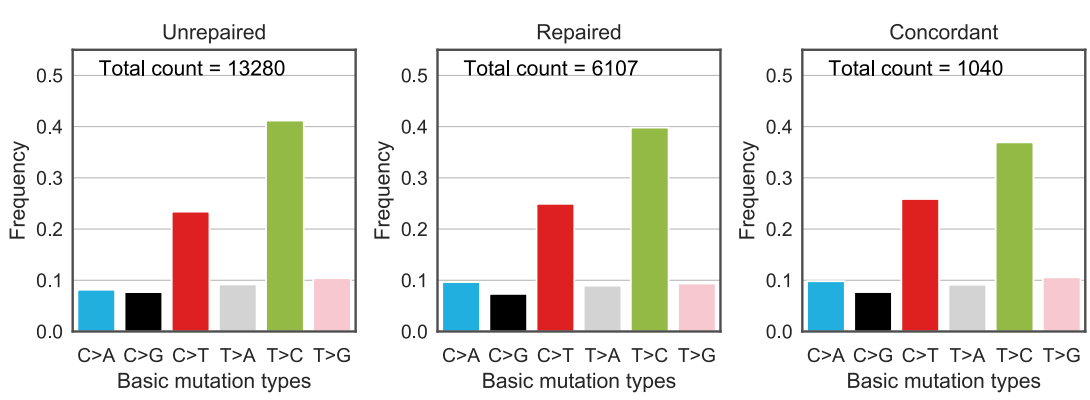

C

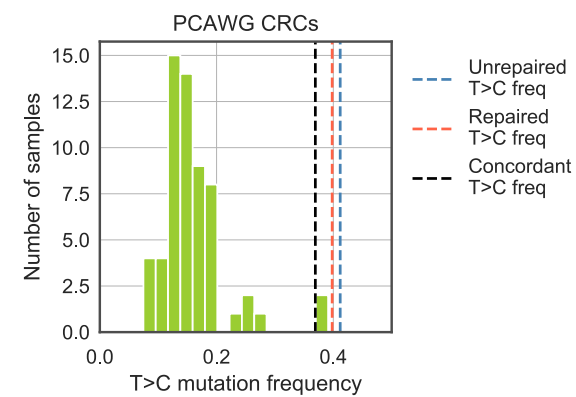

Supplemental Fig7. Mutations from two WGS FFPE CRC samples. (a) Allele frequency versus total reads number of detected variants. The four panels from left to right show mutations detected from unrepaired FFPE, repaired FFPE and concordant mutations in unrepaired and concordant mutations in repaired FFPEs, respectively. Concordant mutations refer to variants are detected in both repaired and unrepaired FFPEs with at least 5 supporting reads. (b) Total count of SBS variants in unrepaired, repaired and concordant mutations. (c) $\mathrm{T}>\mathrm{C}$ mutation frequencies of PCAWG CRC samples. Three dash lines indicate $\mathrm{T}>\mathrm{C}$ mutation frequencies of unrepaired, repaired and concordant mutations from our sequenced FFPE samples. 
a

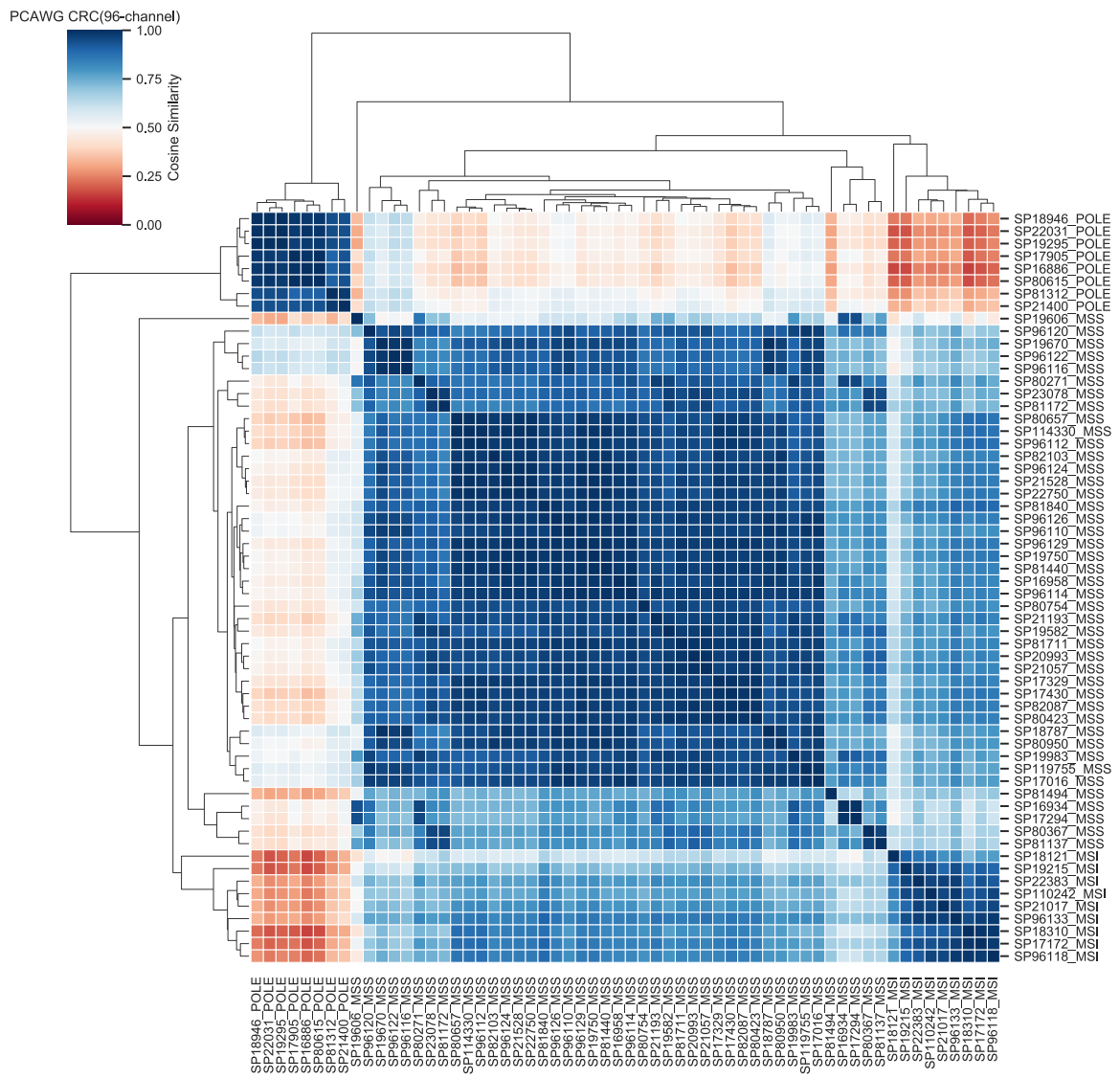

b

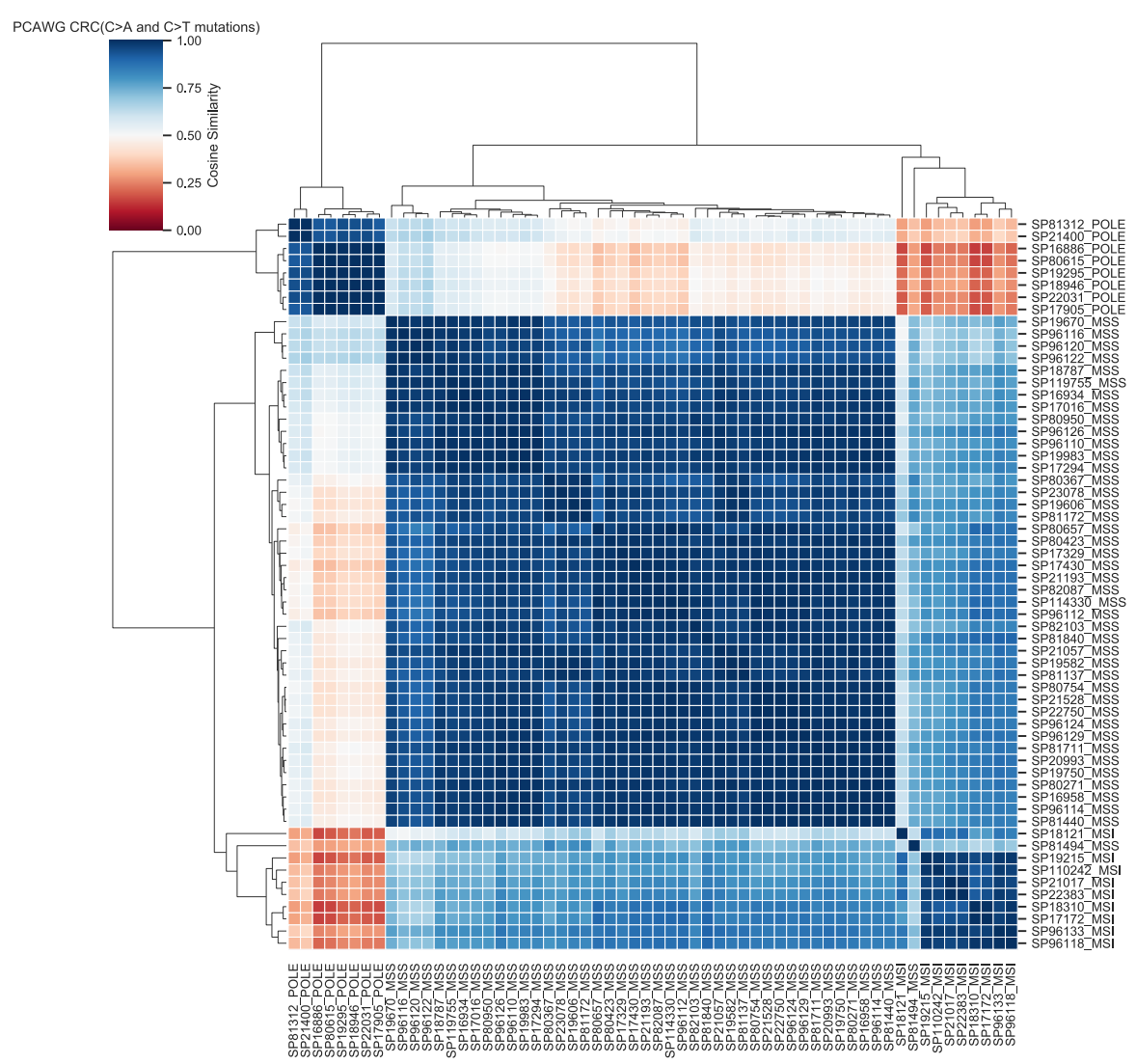

Supplemental Fig8. Clustering PCAWG CRC mutational catalogues. (a) using 96channel profiles. (b) using $\mathrm{C}>\mathrm{A}$ and $\mathrm{C}>\mathrm{T}$ mutation profiles. 
a

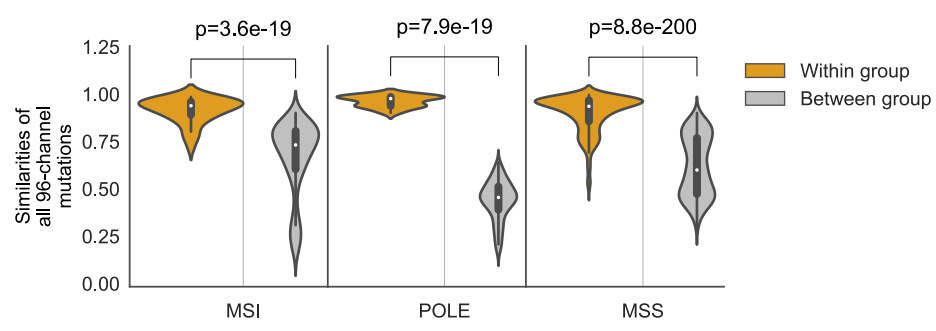

b

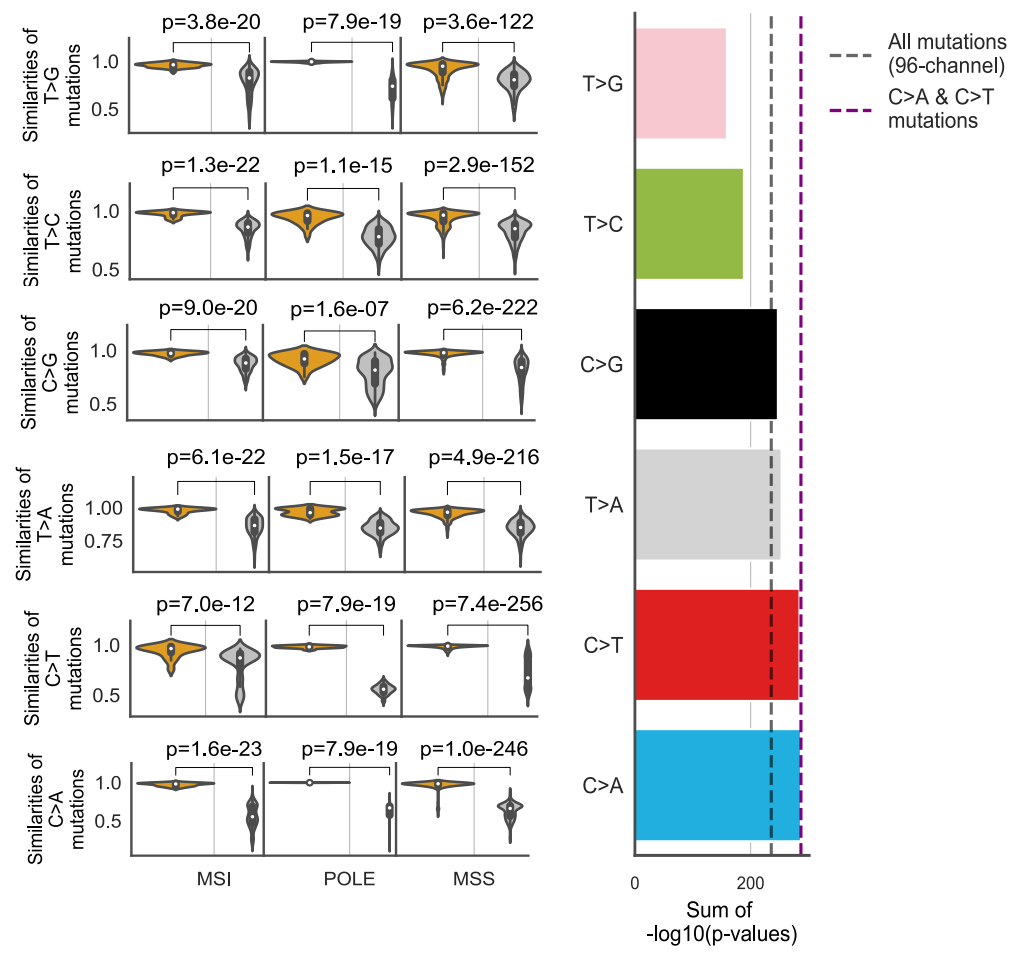

Supplemental Fig9. Comparison of sample-pair similarities within and between subgroups of PCAWG CRCs. PCAWG CRC are grouped based on their known labels, namely POLE, MSI and MSS. (a) Comparison made using full 96 channel mutational profiles. The sample-pair cosine similarities of mutation patterns within and between groups are shown in orange and grey box plot, respectively. The difference for each subgroup is measured by two-sided MannWhitney $U$ test. (b) $\mathrm{C}>\mathrm{A}$ and $\mathrm{C}>\mathrm{T}$ mutation patterns are highly conserved/similar within each subtype. The same comparison in (a) is made but using six basic mutation types separately. We use the sum of $-\log 10$ ( $p$-value) to sort the six mutation types, shown in the right panel. We also use black and purple dash lines to mark sum of $-\log 10$ ( $p$-value) value by using 96-channel and by using $\mathrm{C}>\mathrm{A}$ and $\mathrm{C}>\mathrm{T}$ (32-channel). 

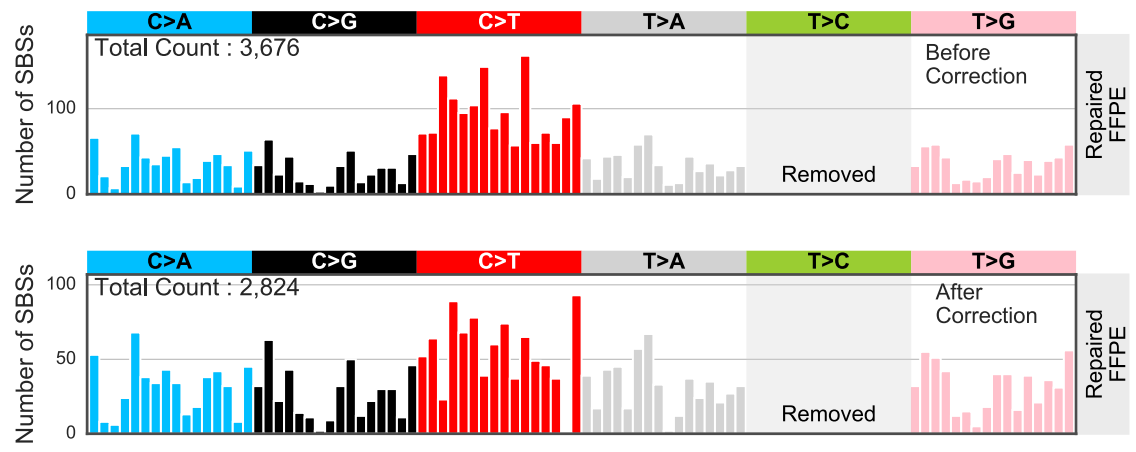

Supplemental Fig10. FFPE noise correction results of repaired FFPE CRC sample. The top panel shows mutational profile before correction. And the lower panel shows the corrected profile. 
a

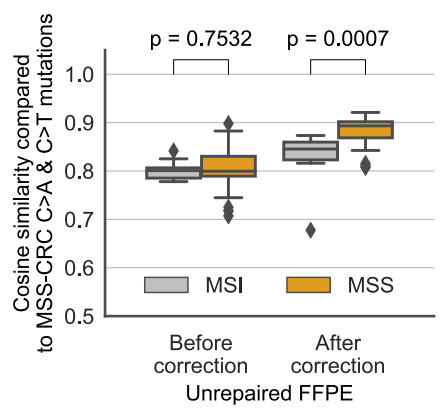

b

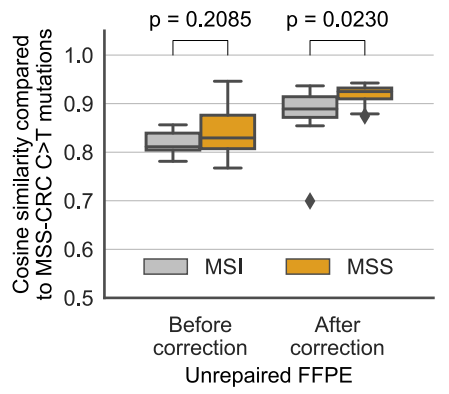

C

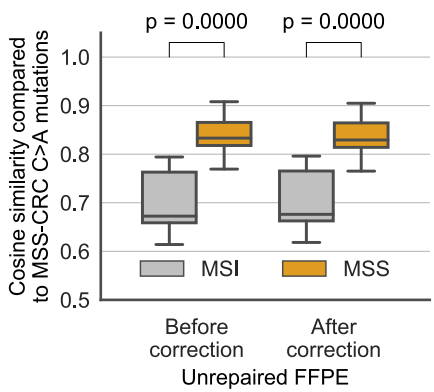

Supplemental Fig11. Correction on unrepaired FFPE CRC sample contributes to classify MSS subtype from MSI. The difference for each subgroup is measured by two-sided MannWhitney $U$ test. (a) Correction makes significant improvement for the classification by using $\mathrm{C}>\mathrm{A}$ and $\mathrm{C}>\mathrm{T}$ mutations. (b) Correction on $\mathrm{C}>\mathrm{T}$ mutations also improves the classification. (c) $\mathrm{C}>\mathrm{A}$ mutation profiles in unrepaired FFPE sample can also be used as classifier. As our correction acts on $\mathrm{C}>\mathrm{T}$ channels mostly, so the $\mathrm{C}>\mathrm{A}$ mutation pattern are almost the same before and after correction (cosine similarity: $\sim 1)$. 


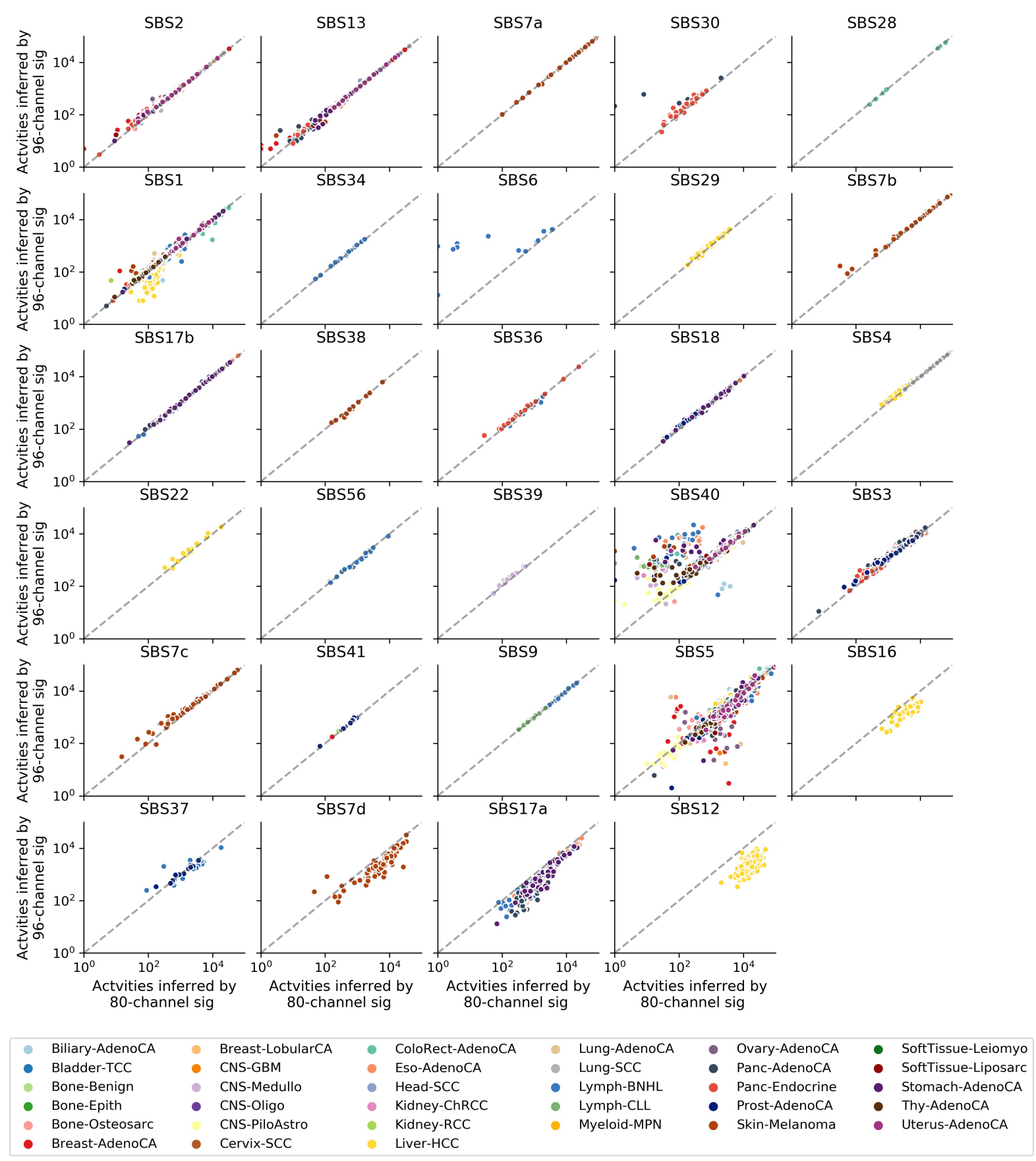

Supplemental Fig 12. Comparison of refitted activity counts using 80-channel and 96-channel signatures for PCAWG data. 
a

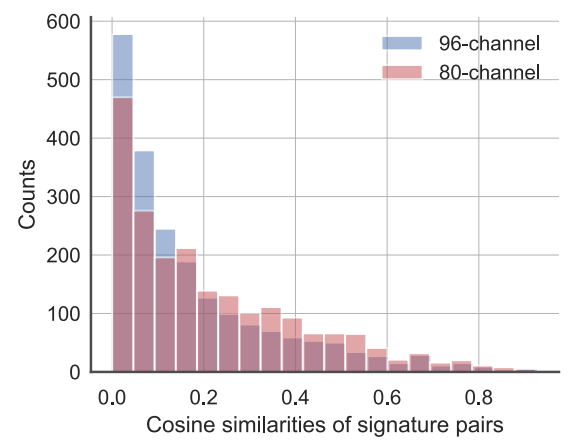

b

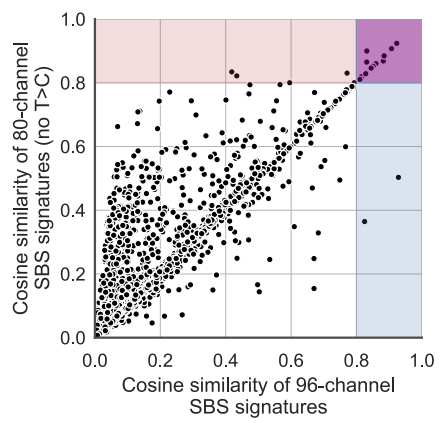

C

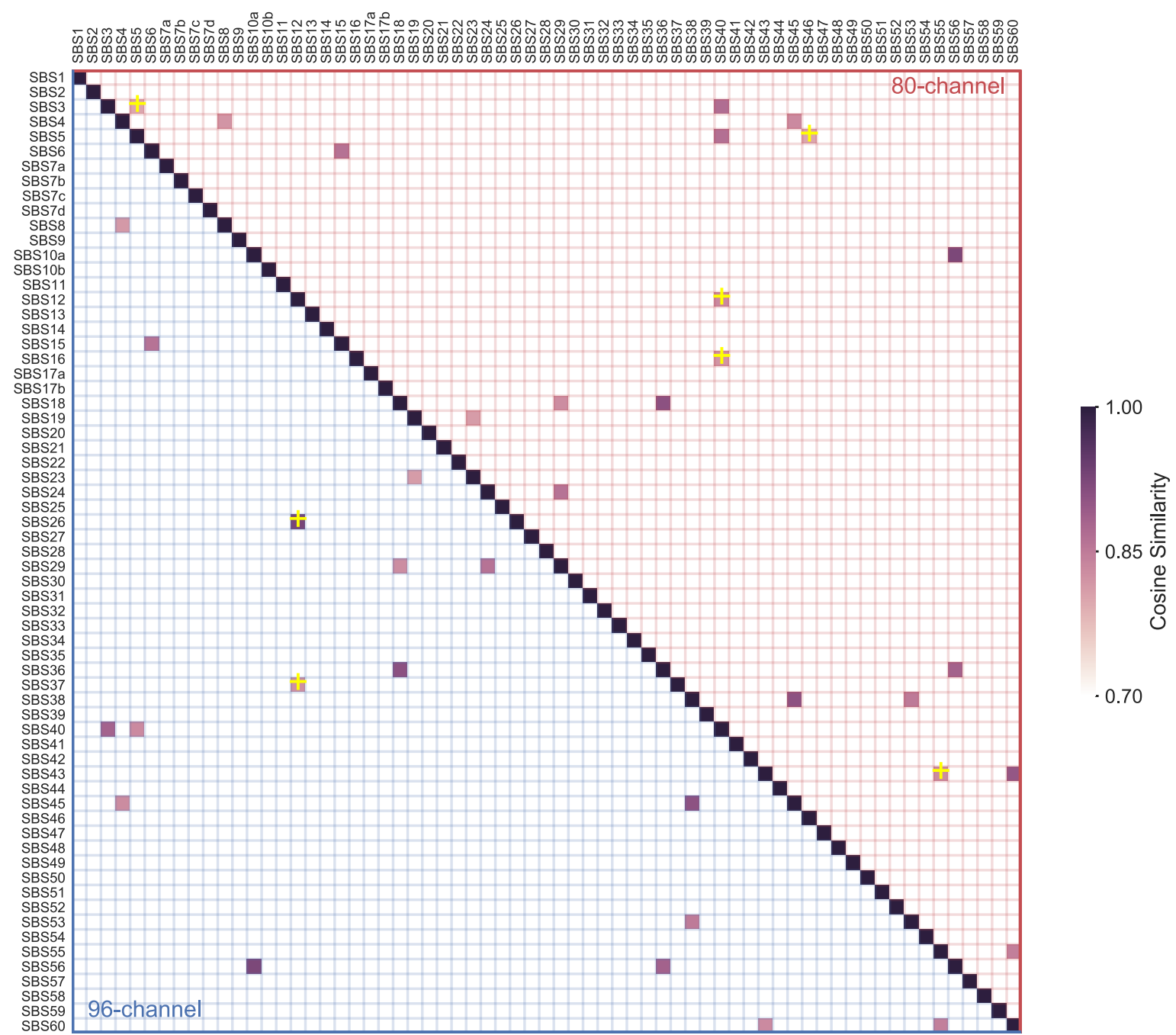

Supplemental Fig 13. Comparison of signature similarities using 96-channel and 80-channel (no T>C) spectra. (a) Histogram of cosine similarities for signature pairs using 96-channel (96c; blue) and 80-channel (80c; pink). (b) Scatter plot of pair-wise cosine similarities using 96c and 80c signatures. Highly similar ( $>0.8)$ signature pairs are highlighted in the plot: 1) purple area shows signature pairs that are highly similar in both signature settings (96c and 80c); 2) blue area contains signature pairs are highly similar by using $96 \mathrm{c}$ profiles, but not highly similar by using $80 \mathrm{c}$; and 3 ) pink area shows pairs with high similarity by using $80 \mathrm{c}$ not $96 \mathrm{c}$. (c) Highly similar signature pairs using $96 \mathrm{c}$ and/or $80 \mathrm{c}$. The upper and lower triangle show the signature pairs calculated using $80 \mathrm{c}$ and $96 \mathrm{c}$, respectively. The signature pair with '+' symbol represents it only exists by using $80 \mathrm{c}$ or by using 96c. The pairs with '+' symbol in upper triangle are the dots from pink area in (b), and those in lower triangle are from blue area in (b). 